\title{
sciendo
}

Int. J. of Applied Mechanics and Engineering, 2021, vol.26, No.4, pp.29-50

DOI: $10.2478 /$ ijame-2021-0048

\section{EFFECTS OF ROTATION ON TRANSIENT FLUID FLOW AND HEAT TRANSFER THROUGH A CURVED SQUARE DUCT: THE CASE OF NEGATIVE ROTATION}

\author{
Mohammad Sanjeed HASAN*, Md. Tusher MOLLAH and Dipankar KUMAR \\ Department of Mathematics, Bangabandhu Sheikh Mujibur Rahman Science and Technology University \\ Gopalganj-8100, BANGLADESH \\ E-mail: sanjeedlhasan@gmail.com \\ Rabindra Nath MONDAL \\ Department of Mathematics, Jagannath University, Dhaka-1100, BANGLADESH \\ Giulio LORENZINI \\ Department of Engineering and Architecture, University of Parma, Parma 43124, ITALY
}

\begin{abstract}
The fluid flow and heat transfer through a rotating curved duct has received much attention in recent years because of vast applications in mechanical devices. It is noticed that there occur two different types of rotations in a rotating curved duct such as positive and negative rotation. The positive rotation through the curved duct is widely investigated while the investigation on the negative rotation is rarely available. The paper investigates the influence of negative rotation for a wide range of Taylor number $(-10 \leq \operatorname{Tr} \leq-2500)$ when the duct itself rotates about the center of curvature. Due to the rotation, three types of forces including Coriolis, centrifugal, and buoyancy forces are generated. The study focuses and explains the combined effect of these forces on the fluid flow in details. First, the linear stability of the steady solution is performed. An unsteady solution is then obtained by time-evolution calculation and flow transition is determined by calculating phase space and power spectrum. When $\operatorname{Tr}$ is raised in the negative direction, the flow behavior shows different flow instabilities including steadystate, periodic, multi-periodic, and chaotic oscillations. Furthermore, the pattern variations of axial and secondary flow velocity and isotherms are obtained, and it is found that there is a strong interaction between the flow velocities and the isotherms. Then temperature gradients are calculated which show that the fluid mixing and the acts of secondary flow have a strong influence on heat transfer in the fluid. Diagrams of unsteady flow and vortex structure are further sketched and precisely elucidate the curvature effects on unsteady fluid flow. Finally, a comparison between the numerical and experimental data is discussed which demonstrates that both data coincide with each other.
\end{abstract}

Key words: flow transition, temperature gradient, unsteady and vortex diagram, experimental validation.

\section{Introduction}

Characteristics of a two-dimensional fluid flow through a rotating curved duct are typically observed not only in mechanical sectors, specifically in chemical reactors, rocket and aircraft engines, air-conditioning, refrigeration, turbo-machinery; but also applied in biological problems such as human lungs and blood circulation in veins and arteries. The analysis of flow through a duct started many years ago, some outstanding reviews on a curved duct are given by Zheng et al. [1] for a pipe, Mondal et al. [2] for a square and a rectangular duct, Yanase et al. [3] for a rectangular duct, Chandratilleke et al. [4] for an elliptical duct. It is investigated that the differences between different types of ducts are characterized based on their force generation as well as the curvature. For example, the curved duct generates two types of force, one is the

\footnotetext{
${ }^{*}$ To whom correspondence should be addressed
} 
centrifugal force that is due to the curvature of the duct and the other one is the Coriolis force that is induced by the duct rotation. The work done due to the centrifugal body force takes place at the outer wall of the duct because of the pressure gradient of the flow. As a result, a two-vortex secondary flow is produced, and this twovortex secondary flow converts into four-vortex under a critical flow condition. These types of vortices were first obtained by Dean [5] and the vortices are called Dean vortices. They are also called hydrodynamic flow instability in the base secondary flow.

Several investigations considered the fluid flow phenomena during a flow through a curved duct. An important phenomenon is the bifurcation together with linear stability and secondary flow characteristics. Two types of bifurcations, such as Pitchfork and Hopf-bifurcations are found to vanish due to an increasing curvature of the duct, see Mondal et al. [6]. Sultana et al. [7] adopted the spectral method to seek out the steady solution branches in the curved rotating duct. The effect of aspect ratios in the steady solution branches was analyzed by Yanase et al. [8]. The influence of the steady and unsteady solution structures in a non-rotating duct for a large aspect ratio was demonstrated by Dolon et al. $[9,10]$. For different values of the equilibrium contact angle, bifurcation structures for partially wetting liquids in the rotational cylinder were studied by Lin et al. [11]. Three-dimensional bifurcation structures of the curved square duct were studied by Watanabe and Yanase [12]. Hasan et al. [13, 14] obtained the two- and four-different steady solution structures of the rotating and non-rotating curved duct, respectively, when the temperature difference was located at the top and bottom walls.

Several researchers investigated the time-dependent behavior by changing the parameters such as the aspect ratio, curvature, Dean number, Grashof number, Taylor number. A detailed explanation of the flow through a curved rectangular duct with unsteady behavior was given by Islam et al. [15], where the temperature was introduced at the bottom wall. The structural change of unsteady behavior between a cylinder and an enclosure was illustrated by Zhang et al. [16]. The unsteady flow structures for different curvatures were conducted by Yanase et al. [17]. The effects of Dean and Grashof numbers in the flow transition were proposed by Mondal et al. [18]. They also drew the phase space to take a clear decision whether the flow is periodic, multi-periodic, or chaotic. Numerical and experimental results of the unsteady solutions were demonstrated by Tsuda and Ohba [19] for a U-shaped duct. Dynamic responses of the curved duct flow for an extensive range of the Dean number, for both the steady asymmetric and symmetric solution branches, were conducted by Wang et al. [20]. Islam et al. [21] showed that the transitional behavior in a rotating curved duct for different values of the Dean number with a wide range of Taylor numbers. They also described the heat transfer effects and fluid mixing. Hasan et al. [22-24] investigated the unsteady flow characteristics as well as the bifurcation structures for both rotating and non-rotating curved ducts. The transition based unsteady solutions and the effects of heat transfer in laminar flow were described numerically by Helal et al. [25].

Characteristics of fluid flow through the curved duct are widely used in metal industry as well as petrol engineering. Two- and three-dimensional studies of fluid flow through the duct were performed by Yanase et al. [26]. Yamamoto et al. [27] presented the secondary flow profiles for a wide range of the Dean and Taylor numbers. A numerical analysis in the secondary flow with convective heat transfer for different aspect ratios (1 to 8) was described by Chandratilleke and Nursubyakto [28]. Mondal et al. [29, 30] compared their numerical results with the experimental data of Chandratilleke et al. [31] and visualized the stream functions and the isotherms for different Dean, Grashof, and Taylor numbers. Datta et al. [32] studied the helical curved duct numerically as well as experimentally. The buoyancy forces in the curved duct are converted into the driving force for the secondary flow which was obtained by Wang et al. [33]. Moreover, it was found that the large secondary velocity created between each of the high-velocity cores. Recently, flow characteristics, heat transfer, and entropy generation were performed by Razavi et al. [34]. Heat transfer in both steady and unsteady solutions, as well as the temperature gradients for both the circular and non-circular ducts were estimated by Hasan et al. [35-37]. Promvonge and Skullong [38] explained the thermal characteristics through the v-shaped duct under a fixed heat-flux condition. Norouzi et al. [39] elaborated first and second normal stresses by enumerating the Nusselt number, and the influence on secondary flow intensity in the elastic property. Garcia et al. [40] analyzed the convection in the rotating spherical cells for the oscillating flows. Chanda et al. [41] showed the heat transfer enhancement due to the unsteady flows 
characteristics in the rectangular duct for both positive and negative rotation. Dolon et al. [42] calculated heat transfer in the stable and oscillating flow behavior for the non-rotating curved duct of large aspect ratio. Facao and Oliveira [43] used the CFD method and fluent code to manipulate heat transfer in the rectangular duct for several Dean numbers. To the best of the authors' knowledge, the physical phenomena of the negative rotation through a curved duct is yet to understand. The paper is helpful to fill up this gap.

The study aims to investigate the unsteady solution and heat transfer for a wide range of negative rotation and between two horizontal sidewalls, respectively. Specifically, the linear stability for negative rotation is discussed, and the time-dependent flow characteristics are investigated through the rotating curved square duct. Furthermore, the change of flow velocity and isotherms in the steady-state, periodic, multiperiodic, and chaotic are observed carefully and the influence of centrifugal, Coriolis, and buoyancy forces in the flow transition is discussed. Moreover, the overall heat transfer through the duct is calculated in order to investigate the combined influence of heating-induced buoyancy force and duct rotation upon the collision of fluid particles.

\section{Physical model and governing equations}

A Newtonian fluid is assumed to be flowing with a fully-developed laminar profile through a curved square duct of constant curvature. The lower wall of the duct is a heating source (red), while the upper wall is a cooling source (sky-blue), the remaining walls are thermally insulated. Figure 1 presents the physical model of the duct, a two-dimension a (2D) cross-section, and the coordinate system with relevant notations. The duct is considered to be rotating about the vertical direction. More details about the physical model description can be found in Hasan et al. [37].

(a)

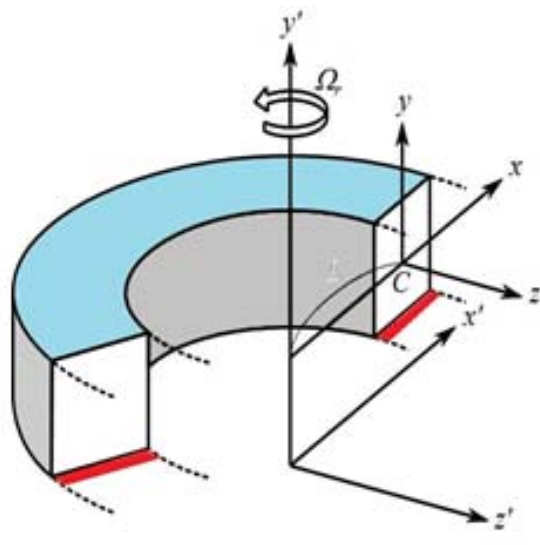

(b)

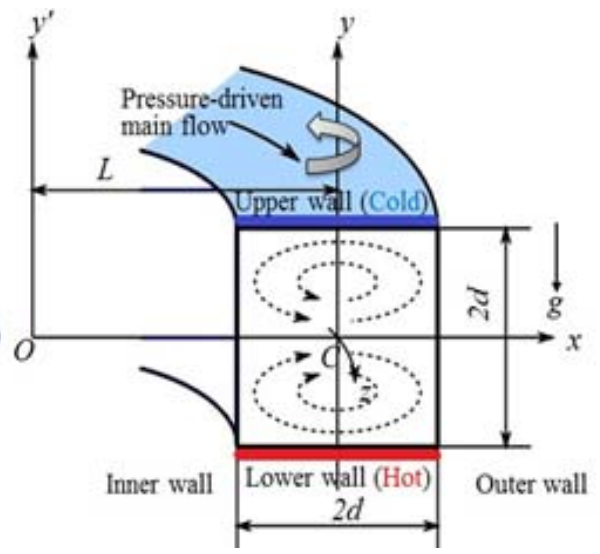

Fig.1. (a) The physical model, (b) 2D cross-sectional view of curved duct.

The continuity equation, Navier-Stokes equations and energy equation in the cylindrical coordinate system are written as,

Continuity equation:

$$
\frac{\partial v_{r}}{\partial r}+\frac{l}{r} \frac{\partial v_{\theta}}{\partial \theta}+\frac{\partial v_{y}}{\partial y}+\frac{v_{r}}{r}=0
$$

Navier-Stokes equations:

$$
\frac{\partial v_{r}}{\partial t}+(v . \nabla) v_{r}-\frac{v_{\theta}^{2}}{r}=-\frac{1}{\rho} \frac{\partial P}{\partial r}+v\left(\tilde{\Delta} v_{r}-\frac{v_{r}}{r^{2}}-\frac{2}{r^{2}} \frac{\partial v_{\theta}}{\partial \theta}\right)
$$




$$
\begin{aligned}
& \frac{\partial v_{\theta}}{\partial t}+(v . \nabla) v_{\theta}+\frac{v_{r} v_{\theta}}{r}=-\frac{1}{\rho r} \frac{\partial P}{\partial \theta}+v\left(\tilde{\Delta} v_{\theta}-\frac{v_{\theta}}{r^{2}}+\frac{2}{r^{2}} \frac{\partial v_{r}}{\partial \theta}\right), \\
& \frac{\partial v_{y}}{\partial t}+(v . \nabla) v_{y}=-\frac{1}{\rho} \frac{\partial P}{\partial y}+v \tilde{\Delta} v_{r}+\beta g T,
\end{aligned}
$$

Energy equation:

$$
\frac{\partial T}{\partial t}+(v \cdot \nabla) T=\kappa \tilde{\Delta} T
$$

Here,

$$
\begin{aligned}
& v . \nabla=v_{r} \frac{\partial}{\partial r}+\frac{v_{\theta}}{r} \frac{\partial}{\partial \theta}+v_{y} \frac{\partial}{\partial y}, \\
& \tilde{\Delta}=\frac{\partial^{2}}{\partial r^{2}}+\frac{1}{r} \frac{\partial}{\partial r}+\frac{1}{r^{2}} \frac{\partial^{2}}{\partial \theta^{2}}+\frac{\partial^{2}}{\partial y^{2}},
\end{aligned}
$$

and $v_{x}, v_{y}$ and $v_{\theta}$ are velocity components in the $r, \theta$ and $y$ axis, respectively. Now, Eqs (2.1)-(2.5) are non-dimensionalized by the assumptions:

$$
\begin{aligned}
& r=L+d x^{\prime}, \quad y=h y^{\prime}, \quad L \theta=-d z^{\prime}, \quad T=T^{\prime} \Delta T, \quad v_{r}=v_{x}=U_{0} u^{\prime}, \quad v_{y}=v_{y}=U_{0} v^{\prime}, \\
& v_{\theta}=-v_{z}=U_{0} w^{\prime}, \quad \rho=\rho_{0} U_{0}^{2} P^{\prime}, \quad G=-\frac{\partial P}{\partial z^{\prime}}, \quad \in=\sqrt{\frac{2 d}{L}}=\sqrt{2 \delta}, \quad \delta=\frac{d}{L} .
\end{aligned}
$$

In this study, $\delta$ is the curvature of the duct identified as the ratio of the width and radius of curvature. The study is based on a two-dimensional flow which is uniform in the $z^{\prime}$-direction and so $\frac{\partial p^{\prime}}{\partial z^{\prime}}=0$. After non-dimensionalization, the transformed Eqs (2.1)-(2.5) can be written as,

Continuity equation:

$$
\frac{\partial u^{\prime}}{\partial x^{\prime}}+\frac{\partial v^{\prime}}{\partial y^{\prime}}+\frac{\delta u^{\prime}}{1+\delta x^{\prime}}+\frac{1}{1+\delta x^{\prime}} \frac{\partial w^{\prime}}{\partial z^{\prime}}=0,
$$

Navier-Stokes equations:

$$
\begin{aligned}
& \frac{\partial u^{\prime}}{\partial t^{\prime}}+\left(v^{\prime} \cdot \nabla^{\prime}\right) u^{\prime}-\frac{1}{2} \epsilon^{2} \frac{w^{\prime 2}}{1+\delta x^{\prime}}=-\frac{\partial P^{\prime}}{\partial x^{\prime}}+\frac{\epsilon}{D n}\left(\tilde{\Delta}_{2}^{\prime} u^{\prime}-\frac{\delta^{2} u^{\prime}}{\left(1+\delta x^{\prime}\right)^{2}}\right), \\
& \frac{\partial v^{\prime}}{\partial t^{\prime}}+\left(v^{\prime} \cdot \nabla^{\prime}\right) v^{\prime}=-\frac{\partial P^{\prime}}{\partial y^{\prime}}+\frac{\epsilon}{D n} \tilde{\Delta}_{2}^{\prime} v^{\prime}+\frac{\beta g \Delta T l}{U_{0}^{2}} T^{\prime},
\end{aligned}
$$




$$
\frac{\partial w^{\prime}}{\partial t^{\prime}}+\left(v^{\prime} . \nabla^{\prime}\right) w^{\prime}+\frac{1}{2} \epsilon^{2} \frac{u^{\prime} w^{\prime}}{1+\delta x^{\prime}}=-\frac{G}{1+\delta x^{\prime}}+\frac{\epsilon}{D n}\left(\tilde{\Delta}_{2}^{\prime} w^{\prime}-\frac{\delta^{2} u^{\prime}}{\left(1+\delta x^{\prime}\right)^{2}}\right)
$$

Energy equation:

where

$$
\frac{\partial T^{\prime}}{\partial t^{\prime}}+\left(v^{\prime} \cdot \nabla^{\prime}\right) T^{\prime}=\frac{\kappa}{d U_{0}} \tilde{\Delta}_{2}^{\prime} T^{\prime}
$$

$$
\left(v^{\prime} \cdot \nabla^{\prime}\right)=u^{\prime} \frac{\partial}{\partial x^{\prime}}+v^{\prime} \frac{\partial}{\partial y^{\prime}}
$$

and

$$
\tilde{\Delta}_{2}^{\prime}=\frac{\partial^{2}}{\partial x^{\prime 2}}+\frac{\partial}{\partial y^{\prime 2}}+\frac{\delta}{1+\delta x^{\prime}} \frac{\partial}{\partial x^{\prime}}
$$

Now, introducing the vorticity vector and the stream function along the $x^{\prime}$ - and $y^{\prime}$-directions, respectively, we have

$$
\begin{aligned}
& \Omega^{\prime}=\frac{\partial v^{\prime}}{\partial x^{\prime}}-\frac{\partial u^{\prime}}{\partial y^{\prime}}=-\frac{1}{1+\delta x^{\prime}}\left(\frac{\partial^{2}}{\partial x^{\prime 2}}+\frac{\partial^{2}}{\partial y^{\prime 2}}-\frac{\delta}{1+\delta x^{\prime}} \frac{\partial}{\partial x^{\prime}}\right) \\
& u^{\prime}=\frac{1}{1+\delta x^{\prime}} \frac{\partial \psi^{\prime}}{\partial y^{\prime}} \quad \text { and } \quad v^{\prime}=-\frac{1}{1+\delta x^{\prime}} \frac{\partial \psi^{\prime}}{\partial x^{\prime}} .
\end{aligned}
$$

Differentiating (2.8) and (2.9) with respect to $x^{\prime}$ and $y^{\prime}$ and subtracting, we have,

$$
\begin{aligned}
& \frac{\partial \Omega^{\prime}}{\partial t^{\prime}}+\left(u^{\prime} \frac{\partial}{\partial x^{\prime}}+v^{\prime} \frac{\partial}{\partial y^{\prime}}\right) \Omega^{\prime}-\frac{\delta u^{\prime}}{1+\delta x^{\prime}} \Omega^{\prime}+\frac{\epsilon^{2} w^{\prime}}{1+\delta x^{\prime}} \frac{\partial w^{\prime}}{\partial y^{\prime}}= \\
& =\frac{\epsilon}{D n}\left(\tilde{\Delta}_{2}^{\prime}-\frac{\delta^{2}}{1+\delta x^{\prime}}\right) \Omega^{\prime}+\frac{\beta g \Delta T l}{U_{0}^{2}} \frac{\partial T^{\prime}}{\partial x^{\prime}} .
\end{aligned}
$$

The simplified equations are found after removing the prime signs from the primitive equations and finally we obtain,

$$
\begin{aligned}
& (1+\delta x) \frac{\partial w}{\partial t}+\frac{\partial(w, \psi)}{\partial(x, y)}-D n+\frac{\delta^{2} w}{1+\delta x}=(1+\delta x) \Delta_{2} w-\frac{\delta}{(1+\delta x)} \frac{\partial \psi}{\partial y} w+\delta \frac{\partial w}{\partial x}-\delta \operatorname{Tr} \frac{\partial \psi}{\partial y} \\
& \left(\Delta_{2}-\frac{\delta}{1+\delta x} \frac{\partial}{\partial x}\right) \frac{\partial \psi}{\partial T}=-\frac{1}{(1+\delta x)} \frac{\partial\left(\Delta_{2} \psi, \psi\right)}{\partial(x, y)}+\frac{\delta}{(1+\delta x)^{2}}\left[3 \delta \frac{\partial^{2} \psi}{\partial x^{2}}-\frac{3 \delta^{2}}{1+\delta x} \frac{\partial \psi}{\partial x}\right]+ \\
& +\frac{\delta}{(1+\delta x)^{2}}\left[\frac{\partial \psi}{\partial y}\left(2 \Delta_{2} \psi-\frac{3 \delta}{1+\delta x} \frac{\partial \psi}{\partial x}+\frac{\partial^{2} \psi}{\partial x^{2}}\right)-\frac{\partial \psi}{\partial x} \frac{\partial^{2} \psi}{\partial x \partial y}\right]-\frac{2 \delta}{1+\delta x} \frac{\partial}{\partial x} \Delta_{2} \psi+ \\
& +w \frac{\partial w}{\partial y}+\Delta_{2}^{2} \psi-G r(1+\delta x) \frac{\partial T}{\partial x}+\frac{1}{2} \operatorname{Tr} \frac{\partial w}{\partial y}
\end{aligned}
$$




$$
\frac{\partial T}{\partial t}+\frac{1}{(1+\delta x)} \frac{\partial(T, \Psi)}{\partial(x, y)}=\frac{1}{\operatorname{Pr}}\left(\Delta_{2} T+\frac{\delta}{1+\delta x} \frac{\partial T}{\partial x}\right)
$$

where

$$
\Delta_{2}=\frac{\partial^{2}}{\partial x^{2}}+\frac{\partial^{2}}{\partial y^{2}} \quad \text { and } \quad \frac{\partial(f, g)}{\partial(x, y)}=\frac{\partial f}{\partial x} \frac{\partial g}{\partial y}-\frac{\partial f}{\partial y} \frac{\partial g}{\partial x}
$$

It is noted that Eqs (2.14)-(2.16) are invariant with respect to the horizontal axis; $D n, G r, T r$ and $\operatorname{Pr}$ are the non-dimensional parameters and addressed as the Dean number, the Grashof number, the Taylor number and the Prandtl number, respectively, defined as

$$
D n=\frac{G d^{3}}{\mu v} \sqrt{\frac{2 d}{L}}, \quad G r=\frac{\beta g \Delta T d^{3}}{v^{2}}, \quad \operatorname{Tr}=\frac{2 \sqrt{2 \delta} \Omega_{T} d^{3}}{v \delta}, \quad \operatorname{Pr}=\frac{v}{\kappa} .
$$

The boundary conditions of the axial velocity $(w)$ and the sectional stream velocity $(\psi)$ are

$$
w( \pm 1, y)=w(x, \pm 1)=\psi( \pm 1, y)=\psi(x, \pm 1)=\frac{\partial \psi}{\partial x}( \pm 1, y)=\frac{\partial \psi}{\partial y}(x, \pm 1)=0
$$

and the boundary conditions for the isotherm $(T)$ are taken as

$$
T(x, 1)=1, \quad T(x,-1)=-1, \quad T( \pm 1, y)=-y .
$$

In the current study, water is the working fluid $(\operatorname{Pr}=7.0)$ and throughout this study we performed numerical simulations for $D n=1000, G r=100$ and the curvature $(\delta)$ and the Taylor number $(\operatorname{Tr})$ vary from $0.001 \leq \delta \leq 0.5$ and $-10 \leq \operatorname{Tr} \leq-2500$ respectively.

\section{Numerical calculation}

\subsection{Numerical processing}

To investigate the flow characteristics together with heat transfer through the curved square duct, the governing equations are codded by using the spectral method (details of the method were given by Gottlieb and Orazag [44]). Expansion of the polynomial functions is one of the main objects of this method. That is, the variables $w, \psi$ and $T$ are expanded in the series of functions $\varphi_{n}(x)$ and $\psi_{n}(x)$ together with the Chebyshev polynomials and the functions are disclosed as,

$$
\left.\begin{array}{l}
\varphi_{n}(x)=\left(1-x^{2}\right) C_{n}(x) \\
\psi_{n}(x)=\left(1-x^{2}\right)^{2} C_{n}(x)
\end{array}\right\} .
$$


Here, $C_{n}(x)$ is the $n^{\text {th }}$ order Chebyshev polynomials defined by $C_{n}(x)=\cos \left(n \cos ^{-1}(x)\right)$. Moreover, $w(x, y, t), \quad \psi(x, y, t)$ and $T(x, y, t)$ are expanded in terms of $\phi_{n}(x)$ and $\psi_{n}(x)$ as:

$$
\begin{aligned}
& \left.w(x, y, t)=\sum_{m=0}^{M} \sum_{n=0}^{N} w_{m n}(t) \phi_{m}(x)\right) \phi_{n}(y) \\
& \psi(x, y, t)=\sum_{m=0}^{M} \sum_{n=0}^{N} \psi_{m n}(t) \psi_{m}(x) \psi_{n}(y) \\
& T(x, y, t)=\sum_{m=0}^{M} \sum_{n=0}^{N} T_{m n}(t) \varphi_{m}(x) \varphi_{n}(y)-y
\end{aligned}
$$

Here, $w_{m n}, \psi_{m n}$ and $T_{m n}$ are the coefficients of expansion which start from 0 for both $m$ and $n$ and end at $M$ and $N$ respectively where $M$ and $N$ are the truncation numbers for the horizontal and vertical axis. After expanding the co-efficient $w_{m n}, \psi_{m n}$ and $T_{m n}$, the obtained values are substituted into Eqs (2.14)(2.16) with applying the collocation method. As a consequence, the nonlinear algebraic equations for $w_{m n}$, $\Psi_{m n}$ and $T_{m n}$ are found. The collocation points are considered as

$$
x_{i}=\cos \left[\pi\left(1-\frac{i}{M+2}\right)\right], \quad y_{i}=\cos \left[\pi\left(1-\frac{j}{N+2}\right)\right]
$$

where $i=1, \ldots, M+1$ and $j=1, \ldots, N+1$. In order to calculate the unsteady solutions, the Crank-Nicolson and Adams-Bashforth methods together with the function expansion (3.2) and the collocation methods are applied to Eqs (2.14)-(2.16).

\subsection{Grid accuracy}

Grid accuracy is checked in this section for several truncation numbers $M$ and $N$. Since the paper describes a square duct flow, so $M$ and $N$ are considered equal. In this study, the values of the resistance coefficient and axial flow negative rotation are taken for several truncation numbers where the parameters of governing equations are fixed $(D n=1000, T r=-1000, G r=100, \delta=0.001)$. Table 1 shows the grid accuracy, and it is observed that the values do not show a substantial change for increasing or decreasing the truncation numbers. To get sufficient accuracy, $M(=N)=20$ has been taken for numerical simulation.

Table 1. Grid accuracy for several truncations numbers.

\begin{tabular}{cccc}
\hline$M$ & $N$ & $\lambda$ & $w(0,0)$ \\
\hline 18 & 18 & .03596529 & 386.001169 \\
20 & $\mathbf{2 0}$ & $\mathbf{. 0 3 5 9 4 6 8 8}$ & $\mathbf{3 8 6 . 0 0 6 2 8 2}$ \\
22 & 22 & .03595800 & 386.062169 \\
24 & 24 & .03595447 & 386.165292 \\
\hline
\end{tabular}




\subsection{Resistance coefficient}

The resistance coefficient, $\lambda$, which is also known as the hydraulic resistance coefficient, is used as the quantity of the flow state in engineering fluids, defined as:

$$
\frac{P_{1}^{*}-P_{2}^{*}}{\Delta_{z}{ }^{*}}=\frac{\lambda}{d_{h}^{*}} \frac{1}{2} \rho\left\langle\omega^{*}\right\rangle^{2} .
$$

The main axial velocity denoted by $\left\langle\omega^{*}\right\rangle$ is calculated as:

$$
\left\langle\omega^{*}\right\rangle=\frac{v}{4 \sqrt{2 \delta d}} \int_{-1}^{1} d x \int_{-1}^{1} \omega(x, y, t) d y .
$$

In this study, $\lambda$ is related to the mean non-dimensional axial velocity $\langle\omega\rangle$ as:

$$
\lambda=\frac{4 \sqrt{2 \delta} D n}{5\langle\omega\rangle^{2}}
$$

where $\langle\omega\rangle=\sqrt{2 \delta d}\left\langle\omega^{*}\right\rangle / v$. Equation (3.6) will be applied to find the resistance coefficient of the flow evolution by numerical calculations.

\section{Results and discussion}

\subsection{Stability analysis}

Here, linear stability is investigated for the flow through a rotating curved duct by assuming $z$ as an independent axis. The function expansion method, together with the collocation method, is applied to the linearized equation for the perturbation of axial flow $(w(x, y))$ and secondary flow $(\psi(x, y))$. To stabilize the unsteady solutions, the perturbation is considered as, $e^{\sigma t}$, where $\sigma=\sigma_{r}+i \sigma_{i}$. Here, $\sigma_{r}$ represents the real value and $\sigma_{i}$ addresses the imaginary value. The solution is linearly stable when all the values of $\sigma_{r}$ are negative. On the other hand, when $\sigma_{r}$ contains at least a single positive value, the solution shows linear instability. It is also noted that the perturbation increases monotonically if $\sigma_{i}$ is equal to zero and oscillatory if $\sigma_{i}$ is not equal to zero. It is observed that there is a strong interaction between the linear stability analysis and the unsteady solutions. Table 2 presents the linear stability analysis where the linear stable points are represented by the bold sign and the consecutive of the linear stable and unstable points are represented by the italic sign. Two different types of flow velocity such as axial $(w)$ and secondary $(\psi)$ flow, and isotherms $(T)$ are shown in Fig.2. for various Taylor numbers. As seen in Fig.2. the axial velocity at the inner wall of the duct is from $\operatorname{Tr}=-10$ to $\operatorname{Tr}=-450$ and the opposite behavior for the axial velocity is found from $\operatorname{Tr}=-500$ which is continued till $\operatorname{Tr}=-2500$. This is caused because of the Coriolis force. On the contrary, the secondary flow velocity shows that two up to six-vortex are created at the outer wall and inner wall of the duct, respectively, from $T r=-10$ to $T r=-450$ and from $\operatorname{Tr}=-500$ to $\operatorname{Tr}=-2500$. It is also seen from the secondary streamlines that the dotted lines have been generated at the lower wall of the duct consecutively and upper wall from $\operatorname{Tr}=-10$ to $\operatorname{Tr}=-450$ and from $\operatorname{Tr}=-500$ to $\operatorname{Tr}=-2500$. This is due to the effect of the Coriolis force, and the number of vortices is increased because of the effects of the duct curvature. It is further remarked that a potential connection is obtained among 
the axial and the secondary velocity. More explicitly, it is observed at $\operatorname{Tr}=-10$ that a pair of high-velocity regions is generated where the upper region is larger than the lower region and a dumbbell is created at the upper region, as a result, an additional two-vortex secondary flow is generated where the upper vortex is larger than the lower vortex because the dumbbell of the axial flow is built at the upper region. The same thing occurred for the other rotational numbers. Temperature profiles show that the densities of isotherm contours are larger where two high-velocity regions of the axial flow and four-, five-, six-vortexes are obtained. So the temperature profiles illustrate that isotherms not only demonstrate the enhancement of heat contained at the duct but also describe the role of fluid mixing.

Table 2. Linear Stability of the negative solution for $\delta=0.001$.

\begin{tabular}{rcccl}
\hline \multicolumn{1}{c}{$T r$} & $\lambda$ & $\sigma_{r}$ & $\sigma_{i}$ & \multicolumn{1}{c}{ Criteria } \\
\hline-10 & .0226198414 & $1.394 \times 10^{1}$ & $2.61 \times 10^{1}$ & Linearly Unstable \\
-182.49 & .0164892486 & $1.109 \times 10^{1}$ & 0 & Linearly Unstable \\
$-\mathbf{- 1 8 2 . 5 0}$ & .0168654054 & $-1.02 \times 10^{-1}$ & 0 & Linearly Stable \\
$-\mathbf{- 3 7 0 . 9 9}$ & .0232515468 & -1.1091 & 0 & Linearly Stable \\
-371.00 & .0232746423 & 4.2531 & 0 & Linearly Unstable \\
-500 & .0272618668 & $1.238 \times 10^{1}$ & $2.337 \times 10^{1}$ & Linearly Unstable \\
& & & & \\
-590.91 & .0293028924 & $1.0345 \times 10^{1}$ & .0346 & Linearly Unstable \\
-590.92 & .0293028924 & -.020139 & 0 & Linearly Stable \\
-912.12 & .0346936717 & -1.1091 & 0 & Linearly Stable \\
-912.13 & .0349693671 & $4.6798 \times 10^{1}$ & $-2.392 \times 10^{1}$ & Linearly Unstable \\
& & & & \\
-1000 & .0359468804 & $7.625 \times 10^{1}$ & $-4.279 \times 10^{1}$ & Linearly Unstable \\
& & & & \\
-1500 & .0411072500 & $1.136 \times 10^{2}$ & $6.568 \times 10^{1}$ & Linearly Unstable \\
-2000 & .0461394361 & $1.359 \times 10^{2}$ & $8.416 \times 10^{1}$ & Linearly Unstable \\
-2500 & .0499782194 & $1.507 \times 10^{2}$ & $1.390 \times 10^{2}$ & Linearly Unstable
\end{tabular}
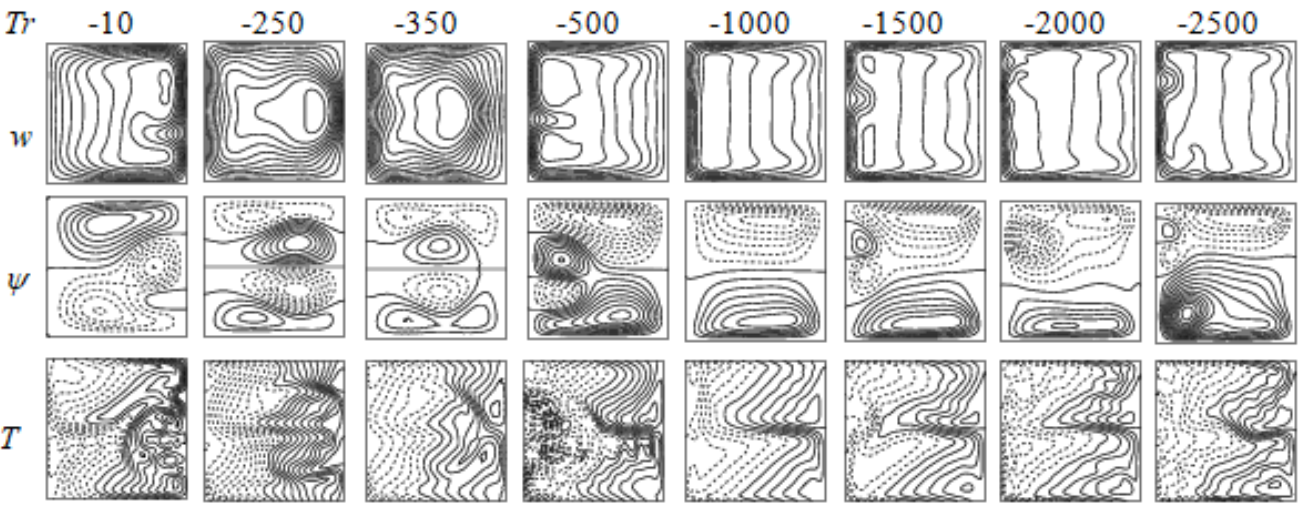

Fig.2. Axial $(w)$ and secondary $(\psi)$ flow, and isotherms $(T)$ for different $T r$. 


\subsection{Time-dependent solutions}

Time-dependent behavior of the unsteady solutions is studied here numerically, where the algorithm of the time-dependent behavior is codded by adopting the Crank-Nicolson and Adams-Bashforth methods alongside the function collocation method. The data is collected from the Code::Blocks, and then the graphs are drawn by using TECPLOT 7. In this paper, the unsteady solutions are investigated for a vast range of negative rotation $(-10 \leq \operatorname{Tr} \leq-2500)$ with the fixed Grashof number $(G r=100)$, curvature $(\delta=0.001)$, and Dean number $(D n=1000)$.

Here, time evolution of the unsteady solution is enumerated for $\operatorname{Tr}=-10$ and then plotted in Taylor number vs. Resistance coefficient plane as shown in Fig.3(a). It is found that the flow characteristics at $T r=-10$ is multi-periodic. To explain the multi-periodic flow behavior, the phase space and power spectrum are drawn. The phase space of the time evolution is depicted in the $\lambda-\gamma$ plane, where $\gamma=\int \psi d x d y$, as shown in Fig.3(b). It is seen that the graph is symmetric about $\gamma=0$ and it has completed multiple orbits before the contour is done. Furthermore, the power spectrum of the unsteady behavior is analyzed in the Frequency vs. Amplitude plane as shown in Fig.3(c). It shows that the line spectrum frequently oscillates for a fixed range of frequency from 0 to 0.4 , where the oscillations become weak when the frequency is higher than 0.25 (approximately). Thus, it can easily be said that the graph presented in Fig.3(a) is absolutely multi-periodic.

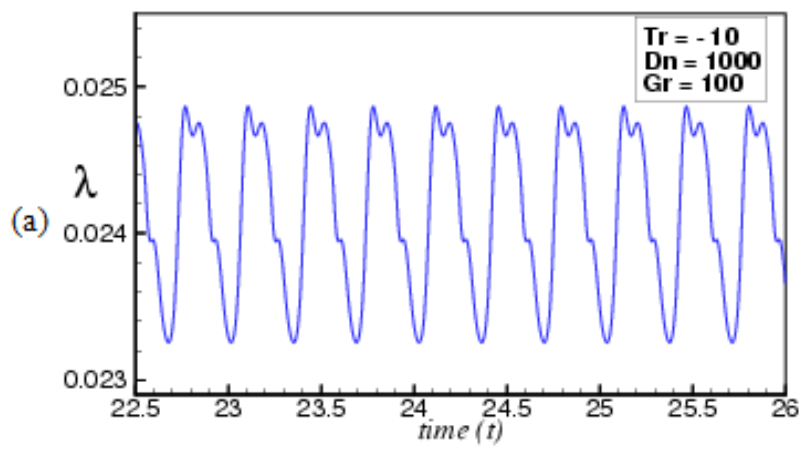

(b)
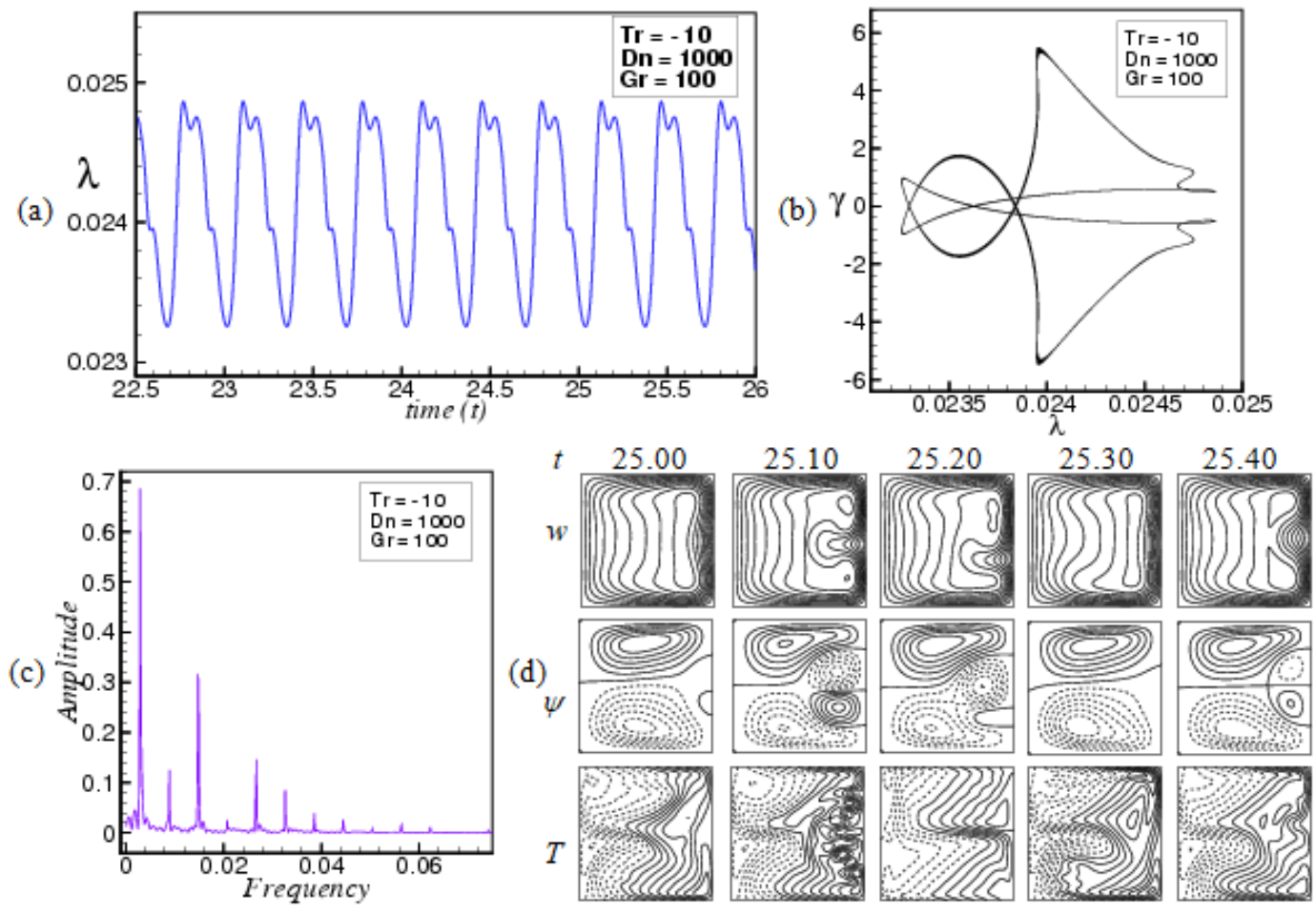

Fig.3. (a) Unsteady flow characteristics, (b) phase space, (c) power spectrum, (d) axial ( $w$ ) and secondary $(\psi)$ flow, and isotherms $(T)$; for $T r=-10$.

To observe vortex generation as well as temperature distribution, streamlines of axial $(w)$ and secondary $(\psi)$ flows, and isotherms $(T)$ are obtained as shown in Fig.3(d). The axial velocity demonstrates that the flow velocity pushes at the inner side of the duct wall and generates low to exalted-velocity regions 
near the outer wall. The secondary flows show that two-, three-, four-vortex solutions are created at the outer wall of the duct, and the isotherms illustrate that the fluid particles are mixed. This is due to the consequence of increasing the temperature and the number of vortexes. It is remarked that there is a strong relationship between the flow velocities and the isotherms. At $t=25.10$, the axial velocity consists of two high-velocity regions and two dumbbells at the top and bottom portions of the duct respectively. As a result, four-vortex stream flow is formed. Again, additional two-vortex structure is observed at the outer wall. It is also seen at $t=25.10$ that the upper dumbbells are larger than the lower one, which dominates the size of the lower vortexes to be smaller than the upper one. Almost a similar trend is observed for secondary flow and isotherms. The isotherms indicate that the density of the streamlines is smaller at the two-vortex solution than the four-vortex. The multi-periodic solution that is investigated by the $2 \mathrm{D}$ calculation in the study is able to specify the presence of three-dimensional (3D) traveling wave solution in real flows which was verified by Mees et al. [45], Wang and Yang [46], and in a recent study by Yanase et al. [8].

If the number of rotation $(\mathrm{Tr})$ of the duct is prolonged in the negative direction, the multi-periodic behavior converts into the steady-state solution, i.e., from $\operatorname{Tr}=-182.50$ the steady-state flow starts and this behavior is continued up to $\operatorname{Tr}=-370.99$, which is also justified by the linear stability analysis (see Table 2). Figure 4(a) represents the steady-state solutions for $\operatorname{Tr}=-250$. Two different types of flow velocity such as axial $(w)$ and secondary $(\psi)$ flow, and isotherms $(T)$ are in Fig.4(b). It is noticed from Fig.4(b) that the flow patterns of the steady-state solution do not change with time.
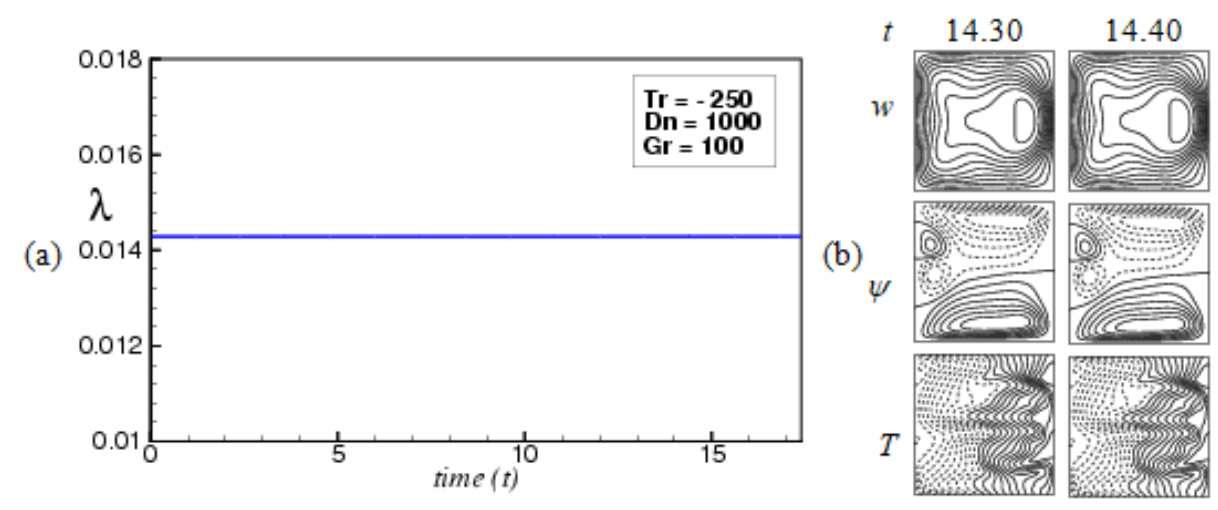

Fig.4. (a) Unsteady flow characteristics, (b) axial $(w)$ and secondary $(\psi)$ flow, and isotherms $(T)$; for $T r=-250$.

If the Taylor number crosses over $\operatorname{Tr}=-370.99$, regular oscillations start again. Figure 5(a) depicts the unsteady behavior for $\operatorname{Tr}=-500$ and it is shown that the unsteady solution is the multi-periodic again. The transition of multi-periodic flow characteristics from one state to another is further explained by phase space and power spectrum analysis as visualized in Figs 5(b) and 5(c). It is demonstrated from the phase space that it has exceeded multiple orbits to reach the origin point. It is also revealed from the power spectrum that the vibration of the line spectrums oscillate till 0.05 and then it weakens gradually. It is further evident from the phase space and power spectrum that the continuation of the secondary flow stream path and the vibration of line spectrums of the frequencies are more unstable than at $T r=-10$. Thus, it can be easily said that the multi-periodic oscillation at $T r=-500$ is stronger than $T r=-10$. Two different types of flow velocity such as axial $(w)$ and secondary $(\psi)$ flow, and isotherms $(T)$ are shown in Fig.5(d). It is observed that the flow velocities display the reverse flow criterion that is found from $\operatorname{Tr}=-10$ to $T r=-500$. It is illustrated that the axial flow velocity produces strong velocity regions at the inner wall where the flow velocity pushes to the outer wall of the duct. The additional two vertices (Ekman vortices) are generated at the inner wall of the duct and the dotted and solid lines have built at the upper and lower wall respectively. This dramatic change of the flow structures occurred because of the Coriolis force of the duct. It is also described from the time variation of the secondary flow that the multi-periodic behaviors have repeated after 70 seconds (see from $t=23.20$ to $t=23.90$ ). 
(a) $\lambda$

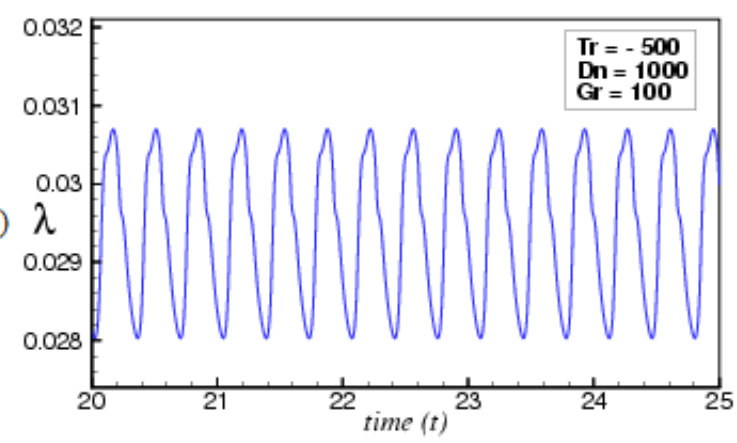

(b)

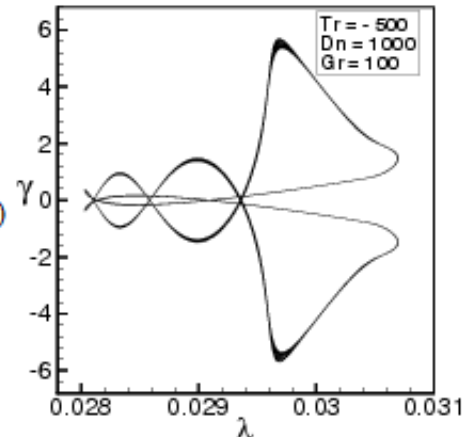

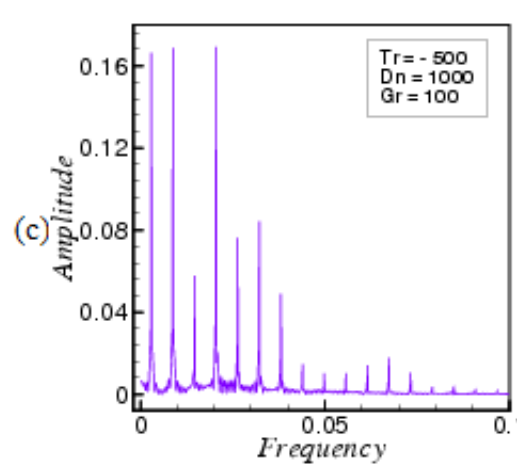
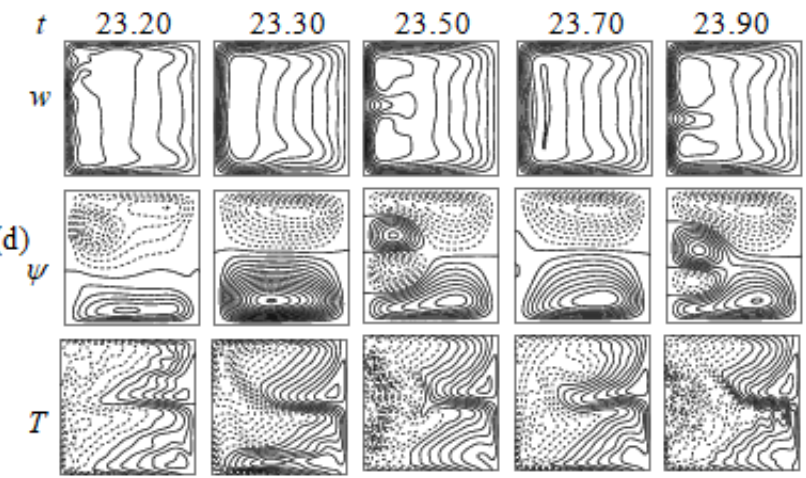

Fig.5. (a) Unsteady flow characteristics, (b) phase space, (c) power spectrum, (d) axial ( $w$ ) and secondary $(\psi)$ flow, and isotherms $(T)$; for $T r=-500$.

(a)

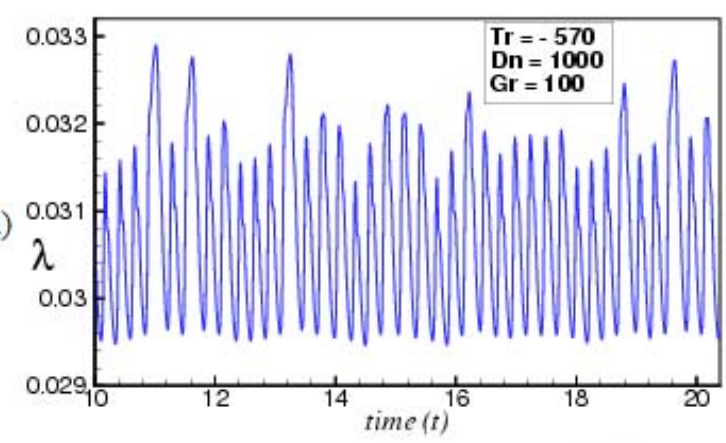

(b)

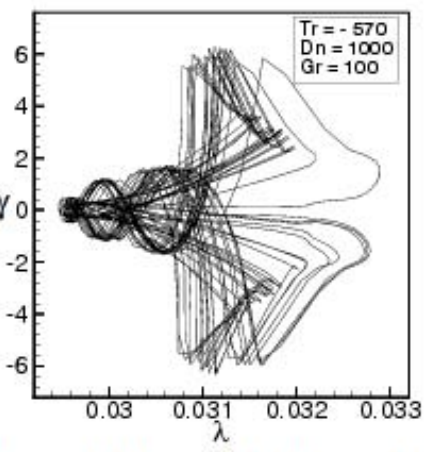

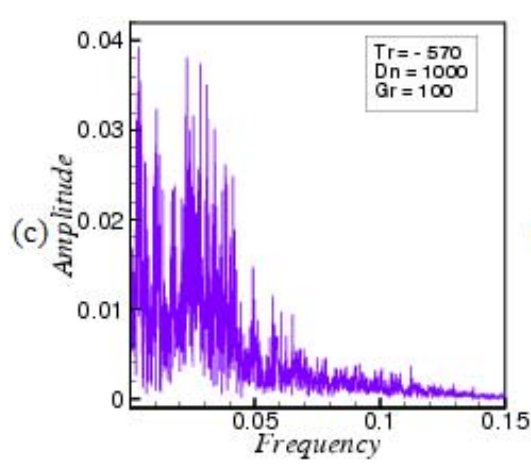
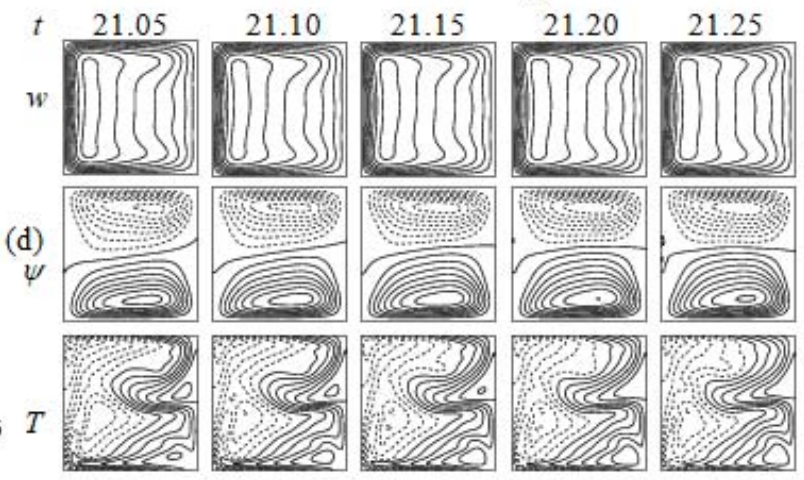

Fig.6. (a) Unsteady flow characteristics, (b) phase space, (c) power spectrum, (d) axial (w) and secondary $(\psi)$ flow, and isotherms $(T)$; for $T r=-570$. 
The multi-periodic oscillation changes to the chaotic oscillation at $\operatorname{Tr}=-570$. Figure 6(a) illustrates the time-dependent behavior of the chaotic oscillation which is also addressed as transitional chaos (detailed in Mondal et al. [6]). The chaotic oscillation is validated with calculating the phase space as well as the power spectrum as depicted in Figs 6(b) and 6(c) consecutively. It is evident that at $\operatorname{Tr}=-570$, the multiple orbits of the phase space, which are continued irregularly and the oscillation period of the line spectrums are larger for $T r=-500$ in both frequency and amplitude axis. Flow velocities, axial $(w)$ and secondary $(\psi)$ flow, and isotherms $(T)$ are illustrated in Fig.6(d). Here, it is seen that there is no high-velocity region in the axial flow, so the secondary flows create only two-vortex solution.

(a)

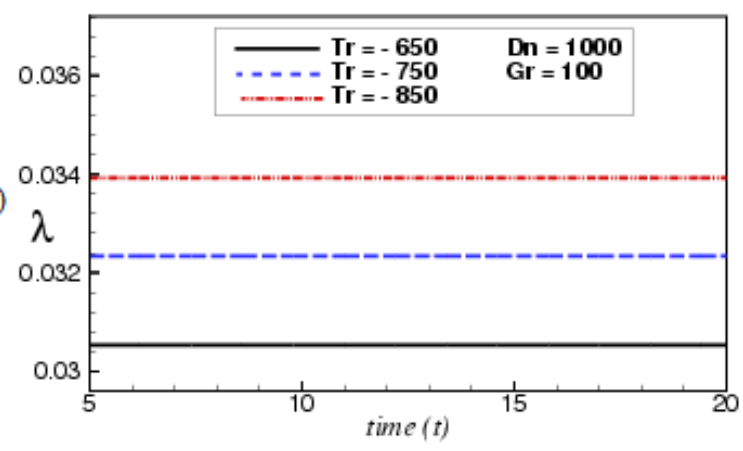

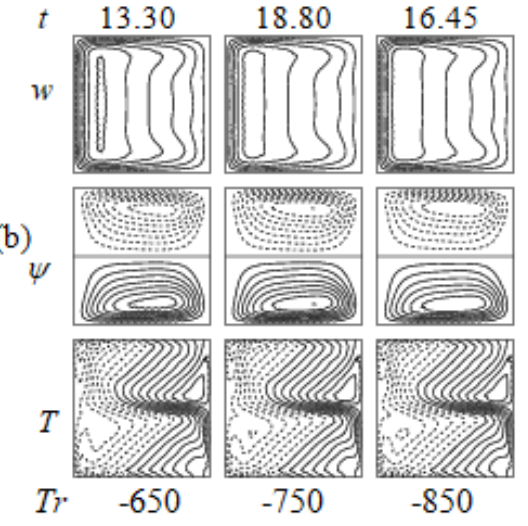

Fig.7. (a) Unsteady flow characteristics, (b) axial $(w)$ and secondary $(\psi)$ flow, and isotherms $(T)$; for $\operatorname{Tr}=-650,-750,-850$.

(a)
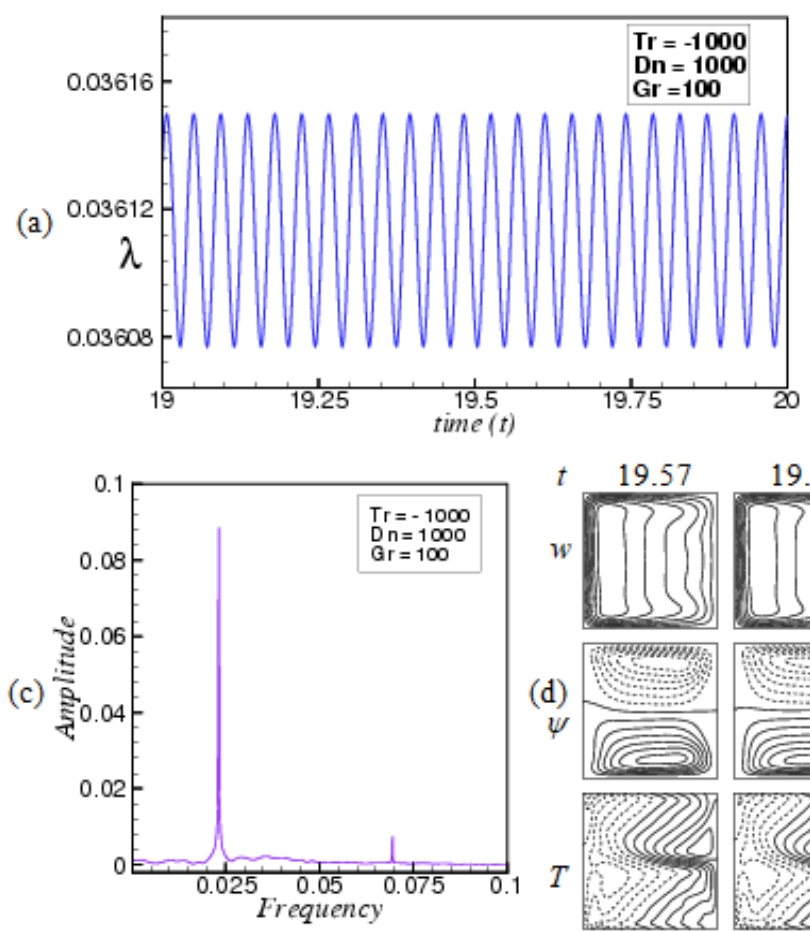

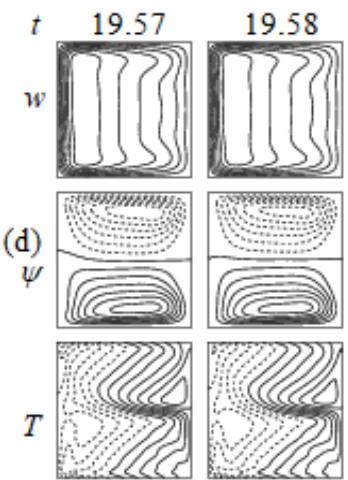

(b)
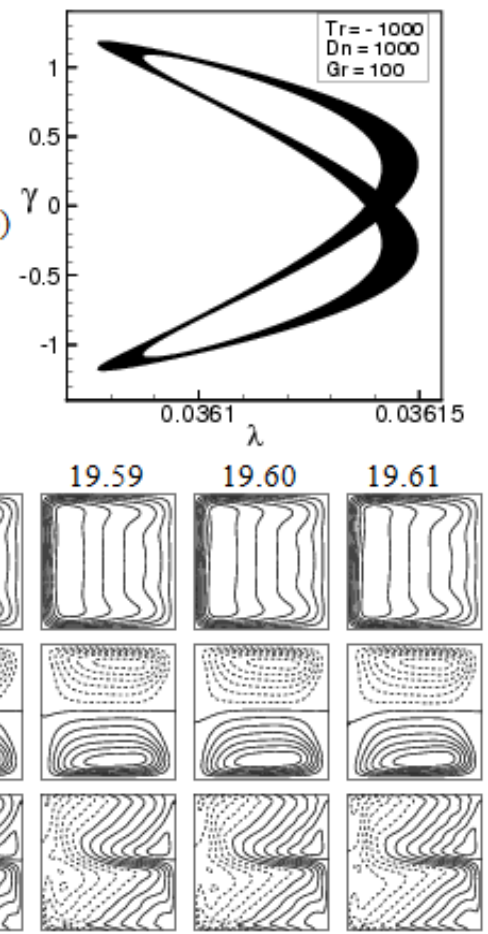

Fig.8. (a) Unsteady flow characteristics, (b) phase space, (c) power spectrum, (d) axial ( $w$ ) and secondary $(\psi)$ flow, and isotherms $(T)$; for $\operatorname{Tr}=-1000$. 
If the Coriolis force speed is increased further in the negative direction, the flow characteristics changes. Figure 7(a) reveals unsteady behavior for $T r=-650,-750,-850$ consecutively and it is seen that the unsteady flow does not oscillate at the required Taylor numbers and that means the flow is in steadystate. Flow velocities, axial $(w)$ and two vortex secondary $(\psi)$ flow, and isotherms $(T)$ are shown in Fig.7(b) for respective Taylor numbers.

Now time history analysis for $\operatorname{Tr}=-1000$ is performed and it is found that the unsteady solution exposes periodic flow behavior as shown in Fig.8(a). The phase space is also represented in Fig.8(b) which describes that it creates a single orbit in the $\lambda-\gamma$ plane. Furthermore, to observe the flow growth more specifically, the power spectrum of the time evolution is calculated as exhibited in Fig.8(c) and it is seen that there are no spectrum lines after oscillating one/single. Thus the phase space and power spectrum indicate that the flow characteristic in Fig.8(a) is certainly periodic oscillation. Flow velocities such as axial (w) and secondary $(\psi)$ flow, and isotherms $(T)$ for periodic solution is exhibited in Fig.8(d) and it is obtained that the unsteady solution exposes only two-vortex asymmetric solutions.

(a)
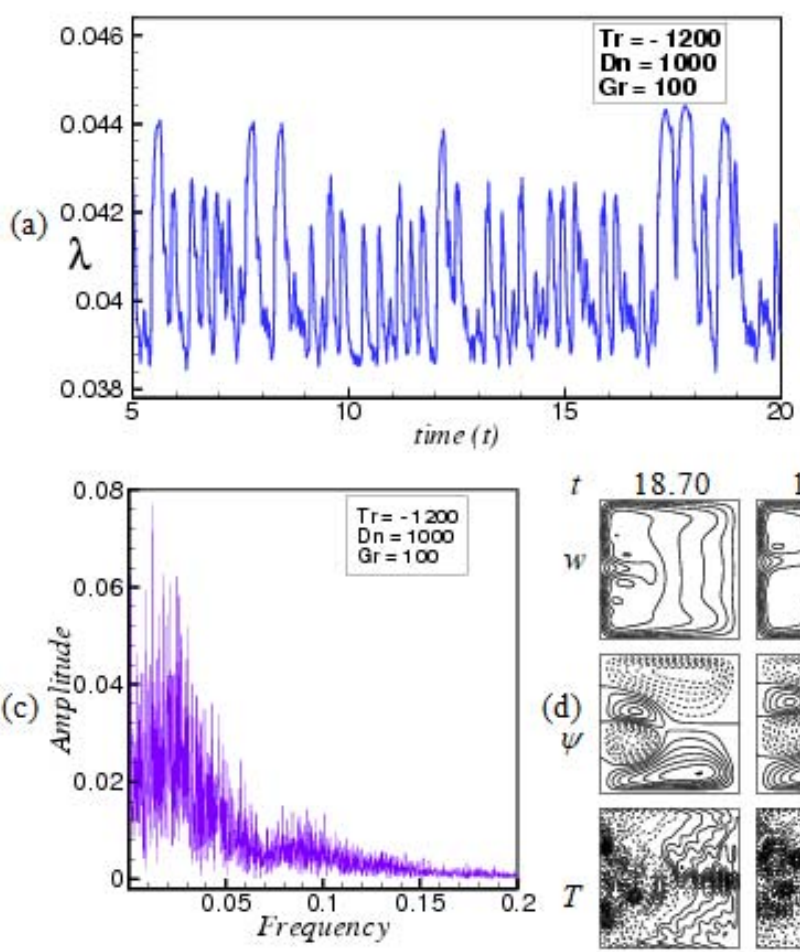

(b)
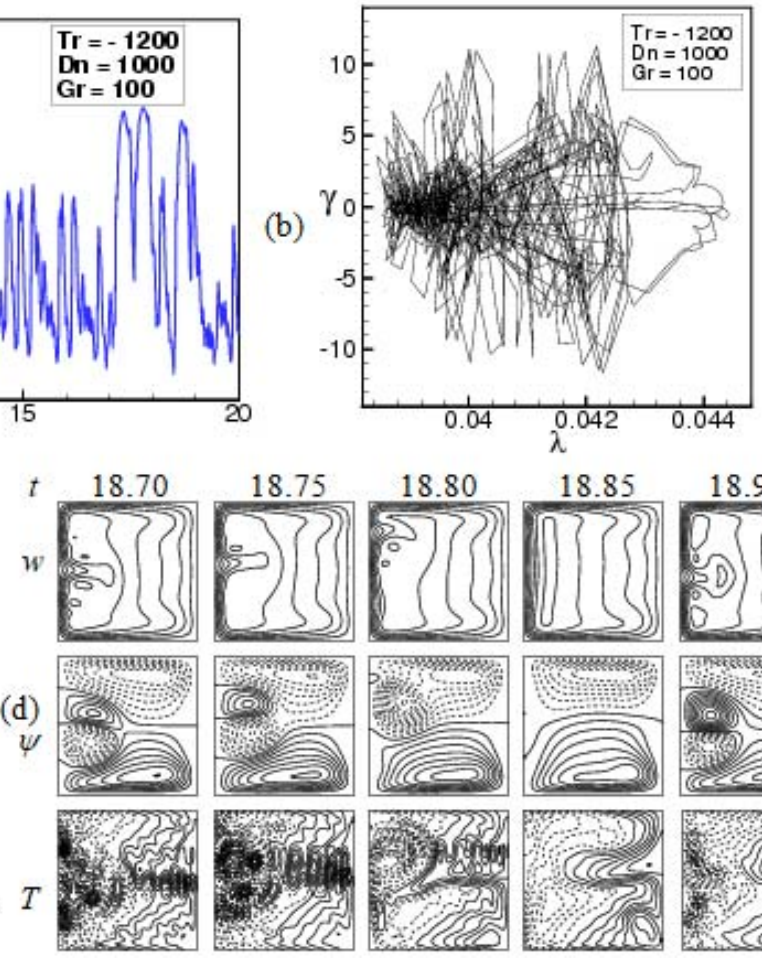

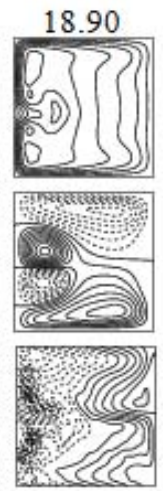

Fig.9. (a) Unsteady flow characteristics, (b) phase space, (c) power spectrum, (d) axial (w) and secondary $(\psi)$ flow, and isotherms $(T)$; for $T r=-1200$.

At $\operatorname{Tr}=-1200$, the regular (periodic) oscillation converts into irregular (chaotic) oscillation as shown in Fig.9(a) and this chaotic oscillation is continued up to $T r=-2500$ as illustrated in Fig.10(a). From the figures, it is evident that the densities of oscillations in the $t-\lambda$ plane have increased for raising the Taylor number. Thus at $T r=-1200$ is designated as transitional chaos and at $T r=-2500$ is termed strong chaos. To justify these irregular oscillations, phase space and power spectrums are enumerated for the respective Taylor numbers as shown in Figs 9(b), 9(c); 10(b), 10(c). It is seen that the region of both phase space and line spectrums have enlarged for rising the rotational number. Axial flow $(w)$, secondary flow $(\psi)$ with two-, three-, four-, five-vortices and temperature profiles $(T)$ are shown in Figs 9(d) and 10(d) 
respectively. The flow characteristics allude that it has allocated symmetrically and the vortices of the secondary flows are formed far away from the outer wall of the duct where the remaining streamlines are not induced by the Dean vortices. It is further observed that due to the increase of the Taylor number in the negative direction, the fluid particles collide and move frequently near the duct walls. At the same time, the fluid particles transfer heat from the duct wall to the fluid. Besides, because of the action of centrifugal, Coriolis, and buoyancy forces in the duct, the fluid particles collide more; as a consequence, the fluids are mixed and this enhances overall heat transfer throughout the fluid and the duct. So it can be easily said that there is certainly a connection between the conversion of the periodic, multi-periodic, and chaotic solution via steady-state solution which is discussed in what follows in detail.

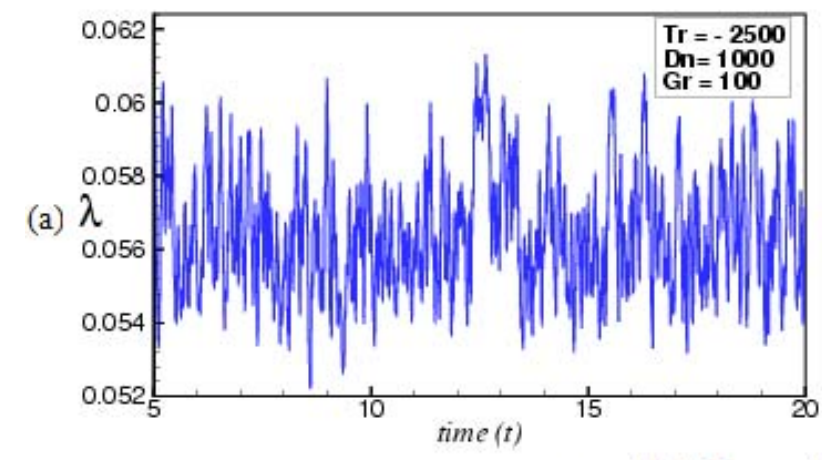

(b)
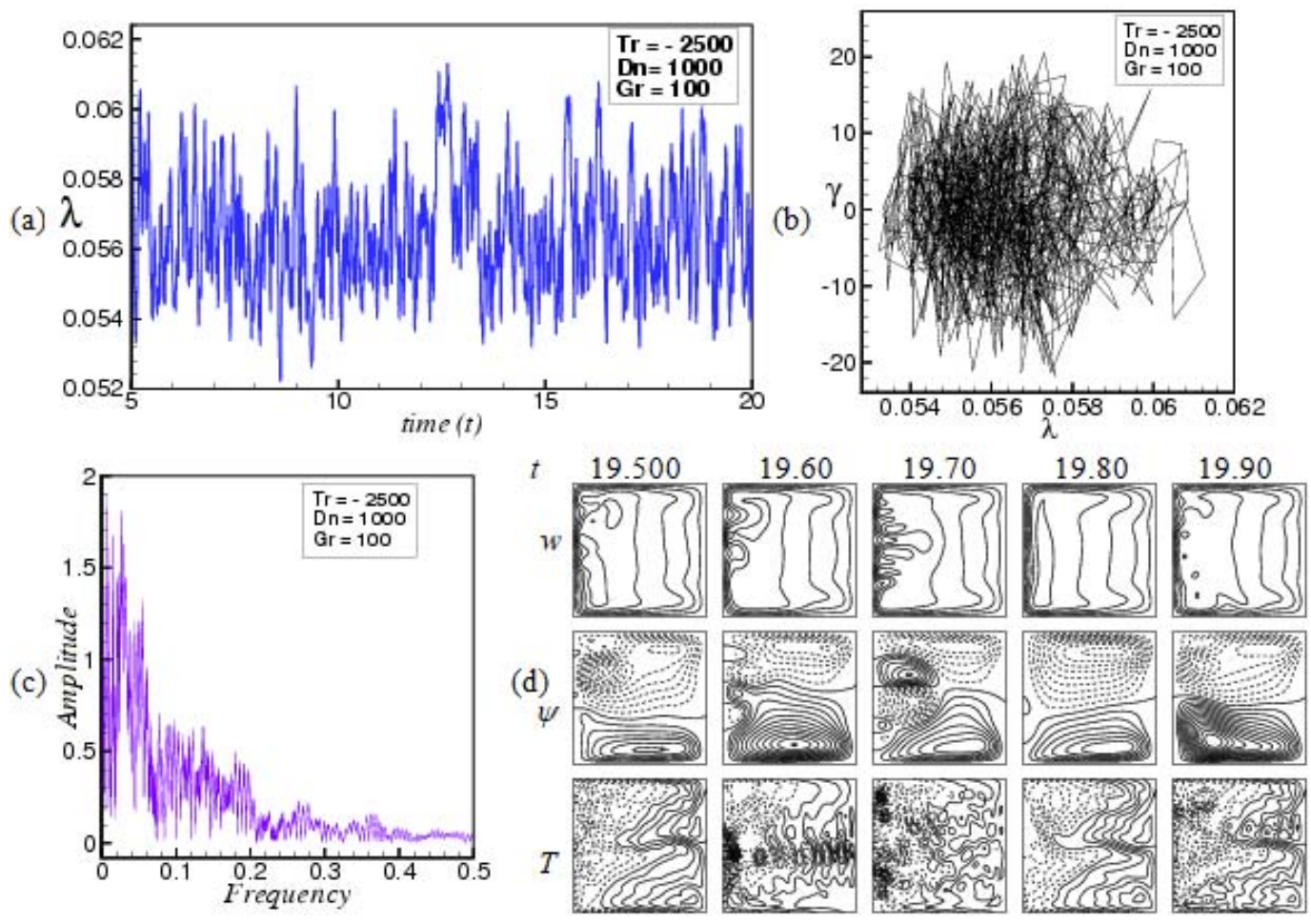

Fig.10. (a) Unsteady flow characteristics, (b) phase space, (c) power spectrum, (d) axial ( $w$ ) and secondary $(\psi)$ flow, and isotherms $(T)$; for $\operatorname{Tr}=-2500$.

\subsection{Temperature gradients}

Here, we obtain the temperature gradients for the heated (bottom) and cooled (top) sidewalls. The influence of Taylor numbers on temperature gradients $\left(\frac{\partial T}{\partial x}\right)$ for cooled and heated sidewalls in the curved square duct is represented in Figs 11(a) and 11(b) respectively. It is seen from Fig.11(a) that $\frac{\partial T}{\partial x}$ reduces at the central region around $y=0$ for an increasing Taylor number. It is also seen from the cooled sidewall that the heat is transferred downwards with vibrations from -1 to -0.5 at the $y$-axis and then it moves upwards from -0.5 to -0.8 and finally it again decreases and at the central of $y=0$, the heat transfer reaches in minimum. The same happens from $y=1$ to $y=0$. Therefore it can easily be said that heat is transferred in the symmetric form on the two sides of the $y=0$. This occurred because two types of forces have acted on it. One is the centrifugal force and the other is 
temperature. Here, because of the mixing of the fluid, the temperature of the duct becomes high. On the other hand, after decreasing of $\frac{\partial T}{\partial x}$ from two sides of -1 to -0.3 and 1 to 0.3 at the $y$ plane respectively, for heated sidewalls, $\frac{\partial T}{\partial x}$ increases monotonically at the central region of $y=0$. It is clearly seen that $\frac{\partial T}{\partial x}$ is enriched by the secondary flow not only in the central region but in other regions within the required range.

(a)

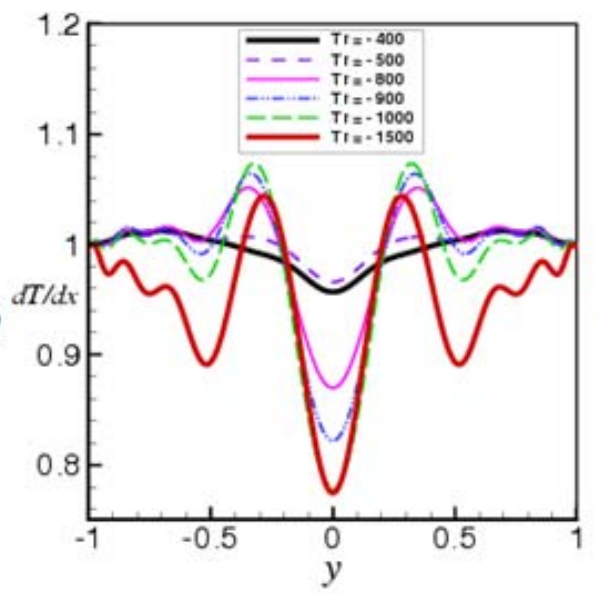

(b)

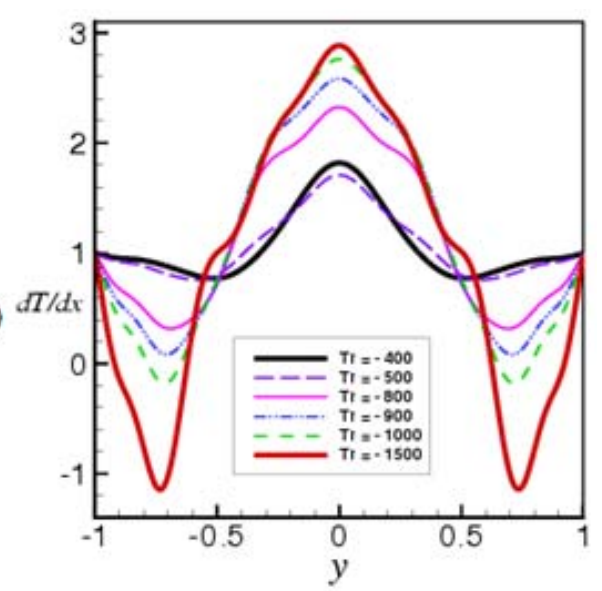

Fig.11. Temperature gradients for $D n=1000, G r=100, \delta=0.001$, (a) cooled sidewall, (b) heated sidewall.

\subsection{Time evolution and vortex diagram}

(a)

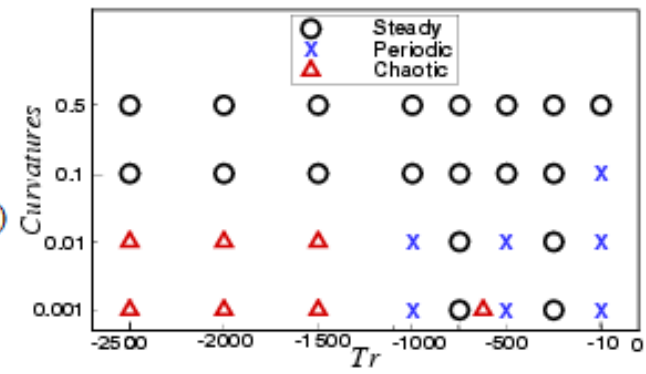

(b)

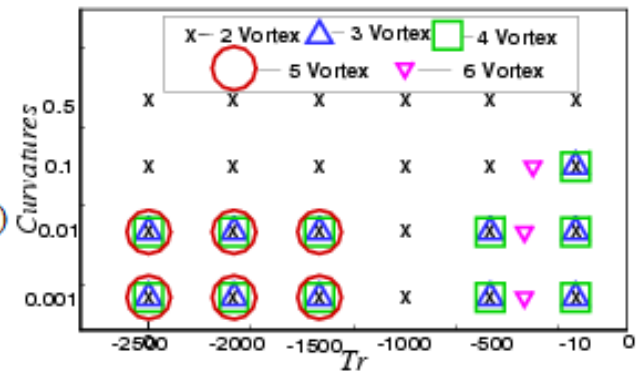

Fig.12. (a) unsteady, (b) vortex diagram of secondary flows; for negative rotation at $0.001 \leq \delta \leq 0.5, D n=1000$ and $G r=100$.

The time evolution diagram is prezented in Fig.12(a) for a wide range of negative rotation $(-10 \leq T r \leq-2500)$ and curvature $(0.001 \leq \delta \leq 0.5)$. In Fig.12(a), circle, cross, and triangles represent the steady-state, periodic/multi-periodic, chaotic solution respectively. It is known that the $T r-\delta$ plane that nearly all types of flow behaviors such as steady, periodic, multi-periodic and chaotic solutions are found at $\delta=0.001$. Almost the same behavior is obtained except $T r=-570$, i.e., for increasing the curvature the chaotic behavior is diminished from that Taylor number. A dramatic change is obseved from $\delta=0.001$ to $\delta=0.1$. Different types of flow characteristics are found for increasing the curvatures. At $\delta=0.1$, only periodic behavior is obtained for $T r=-10$ which is also converted to steady-state at $\delta=0.5$. So it can easily be said that the flow characteristics depend not only on the Coriolis force but also on the centrifugal force. On the other hand, secondary flow structures are also observed for various Taylor numbers as shown in Fig.12(b). It is seen that two-, three-, four-, five-, six- vortex solutions are obtained for different Taylor numbers. In the diagram, two-, three-, four-, five-, six- vortex are designated by the cross, triangle, square, circle, and diamond respectively. Two 
different comments can be made on the diagram. The first one is, the number of vortices has increased at regular and irregular oscillation more than the steady-state oscillation except for $\operatorname{Tr}=-250$. The other is, the number of vortices has diminished for increasing the curvature of the duct. More explicitly, two up to six vortexes secondary flow is generated in $\delta=0.001$ and $\delta=0.01$ whereas two up to four vortex solutions are obtained for $\delta=0.1$, and for increasing the curvature more, only two vortex solutions are created for $\delta=0.5$.

\subsection{Numerical vs. experimental validation}

The numerical studies are also compared with the experimental results to verify the accuracy. The verification is performed not only for the rotational duct but also for the non-rotational duct.

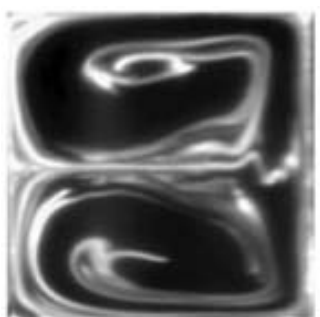

$D n=153$

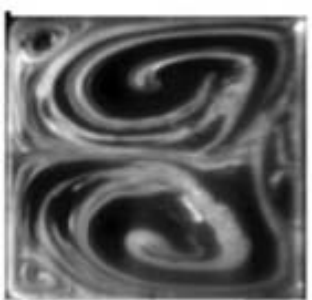

$D n=73$

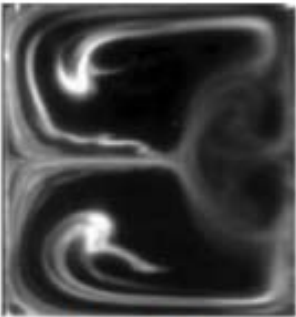

$D n=176$

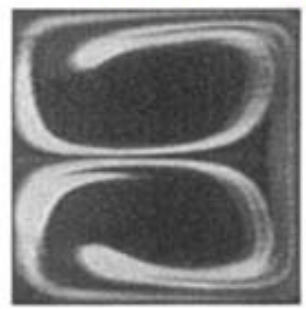

$D n=125$

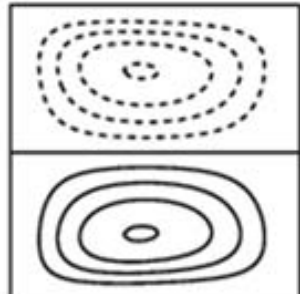

(a) $D n=148$

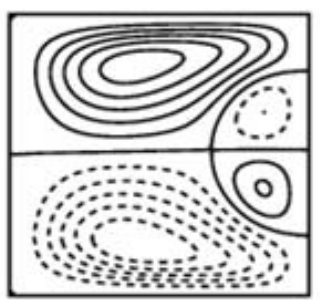

(c)

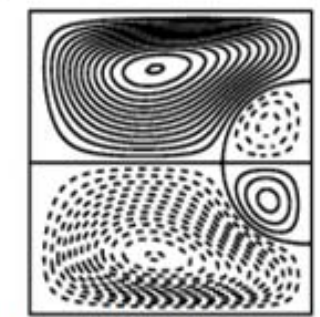

(e)

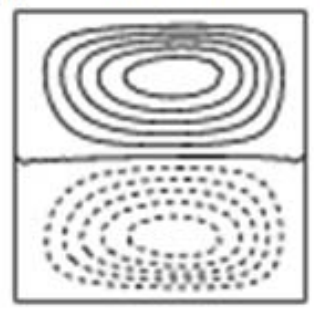

(g)

$D n=117$ (iv)

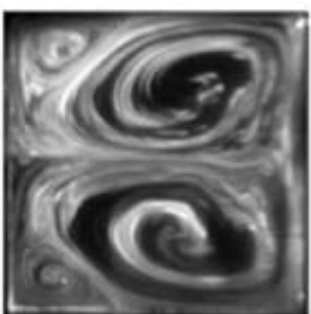

(i)

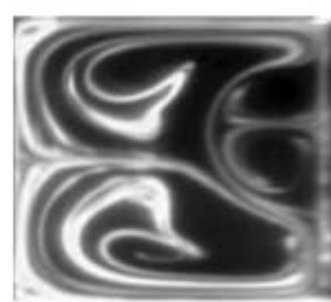

(ii)

$D n=142$

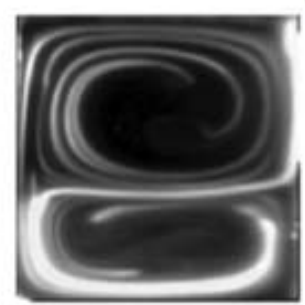

(iii)

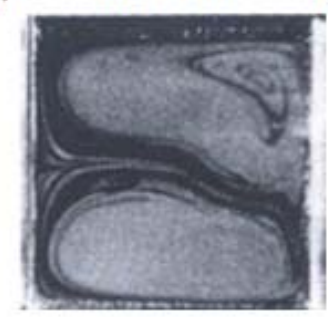

$D n=300$

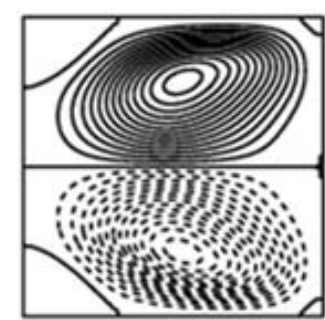

(b)

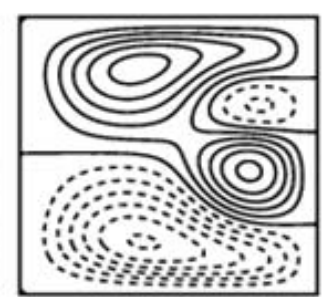

(d) $\quad D n=138$

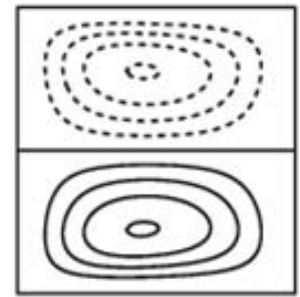

(f) $D n=89$

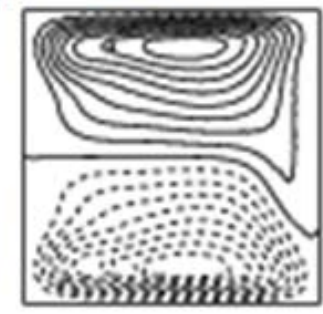

(h) $D n=310$

Fig.13. Experimental (left) vs. numerical (right) results; (i-iii) rotating curved duct by Yamamoto et al. [47], (a-d) $\operatorname{Tr}=-150$, (e-f) $\operatorname{Tr}=150$; (iv) non-rotating curved duct by (g) Wang and Yang [46], (h) Bara et al. [48].

Figure 13 shows a comparison of the secondary flow of numerical and experimental data. The left side of the figures shows the experimental data where the right side exposes the numerical outcome. The experimental results of Figs 13(i) and 13(ii) represent negative and positive rotation successively which is 
obtained by Yamamoto et al. [47] and Figs 13(iv) and 13(v) illustrate the non-rotating case studied by Wang and Yang [46] and Bara et al. [48] respectively. Yamamoto et al. [47] presented experimental data for curved duct of square cross section for both positive and negative rotation. They used a water flow tank where the dye was injected continuously and this dye and alcohol solution had the same weight as water. Firstly, they fixed the duct rotation $(T r=150)$ in the positive direction for several Dean numbers and took photographs at an angle of $180^{\circ}$ inlet. The same work was conducted for the fixed negative rotation $(\operatorname{Tr}=-150)$. In our investigation, we change parameters to obtain the same results as Yamamoto et al. [47].

After plotting the secondary flow, it is seen from Figs 13(i), (ii) and (iii) that the numerical figures of secondary flow are the same as the experimental outcomes. Experimental results for the non-rotating duct are given by many scholars. The experimental data in Figs 13(iv) and 13(v) were also considered by Wang and Yang [46] and Bara et al. [48] for the non-rotating curved duct. Wang and Yang obtained their experimental data by visualization technique at $270^{\circ}$ inlet with curvature ratio 0.4. Bara et al. [48] experimented with injecting smoke through the duct and took a photo at $240^{\circ}$ inlet. Here, we take $T r=0$ for the non-rotating duct, and change the numerical parameters with the same as the experimental data. It is obtained that the numerical outcomes found by the authors are evidently similar to the experimental data.

\section{Conclusions}

A computation based model for the curved rotating duct is presented and the fluid flow characteristics through the duct are investigated for a wide range of values of the Taylor number in the negative direction $(-10 \leq \operatorname{Tr} \leq-2500)$. After an extensive survey, the following outcomes are achieved:

- Firstly, the linear stability for negative rotation demonstrates that the flow is linearly stable in two different regions of Taylor number $(-182.50 \leq T r \leq-370.99$ and $-590.92 \leq T r \leq-912.12)$.

- Time-dependent solutions of the unsteady flow illustrate that the flow transition undergoes different flow instabilities, "multi-periodic $\rightarrow$ steady-state $\rightarrow$ multi-periodic $\rightarrow$ chaotic $\rightarrow$ steadystate $\rightarrow$ periodic $\rightarrow$ chaotic" and it is also obtained that the flow transitions become stronger, if the Taylor number is raised in the negative direction. A strong connection between the linear stability and the unsteady behavior is found, i.e., the unsteady solution totally satisfies the linear stability analysis.

- Two different flow velocities such as axial and secondary flow and the isotherms are given for various Taylor numbers. We obtained that the axial velocity impulses to the inner wall and two up to six vortex solutions are generated at the exterior wall of the duct from $\operatorname{Tr}=-10$ to $\operatorname{Tr}=-250$ approximately. For $\operatorname{Tr}=-300$ to $\operatorname{Tr}=-2500$, on the other hand, the axial velocity pushes to the exterior wall of the duct and two up to five vortex solutions are generated at the inner wall of the duct. This change of flow structures is affected by the Coriolis force and the centrifugal force of the duct. A relation between the axial flows and the secondary flows is also developed in study. The isotherms for both cases illustrate that the fluid has mixed because of the rotation, as a result, the number of vortices has increased more at the regular and irregular oscillation and it has enhanced total heat transfer of the fluid.

- The unsteady flow and the vortex diagram illustrate that for increasing the curvature of the duct the oscillating behavior and the number of vortices decreases.

- Finally, the numerical results are analyzed with the experimental data and it is observed that numerical computations totally agree with the experimental outcomes.

\section{Nomenclature}

$$
\begin{aligned}
D n & - \text { Dean number } \\
d & - \text { half width of the cross section } \\
G r & - \text { Grashof number }
\end{aligned}
$$




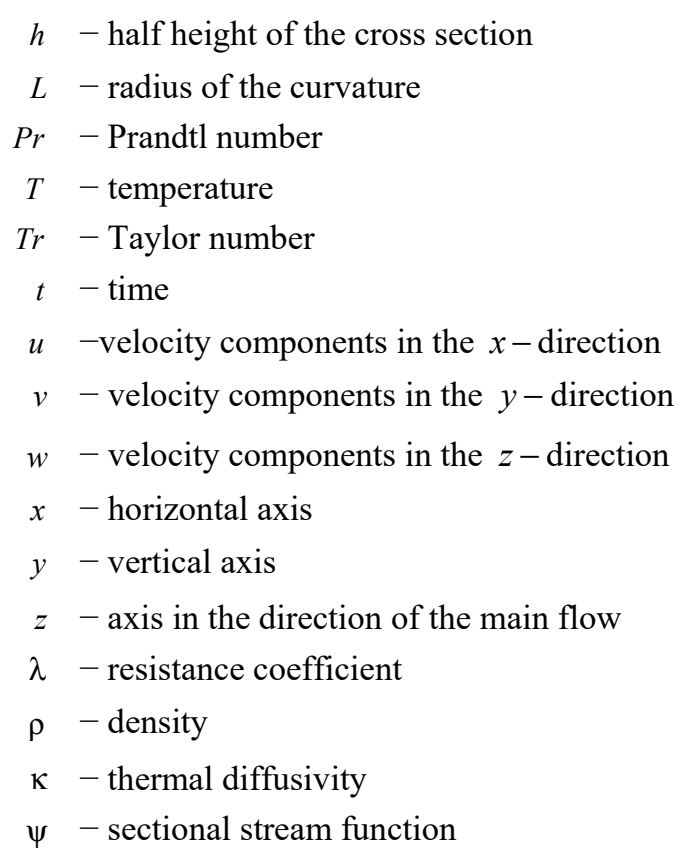

\section{Acknowledgement}

The authors are very much grateful to the unanimous reviewers as well as the journal who have spent their valuable time to review the article fast and helped us to improve the quality and comprehensiveness of the article with constructive comments.

\section{References}

[1] Zheng Y., Jiang P.X., Luo F. and Xu R.N. (2019): Instability during transition to turbulence of supercritical pressure $\mathrm{CO}_{2}$ in a vertical heated serpentine tube.- International Journal of Thermal Sciences, vol.45, Article No.105976. https://doi.org/10.1016/j.ijthermalsci.2019.105976

[2] Mondal R.N. (2006): Isothermal and Non-isothermal Flows through Curved ducts with Square and Rectangular Cross Sections.- Ph.D. Thesis, Department of Mechanical and Systems Engineering, Okayama University, Japan.

[3] Yanase S., Mondal R.N. and Kaga Y. (2005): Numerical study of non-isothermal flow with convective heat transfer in a curved rectangular duct.- International Journal of Thermal Science, vol.44, No.11, pp.1047-1060. https://doi.org/10.1016/j.ijthermalsci.2005.03.013

[4] Chandratilleke T.T., Nadim N. and Narayanaswamy R. (2013): Analysis of secondary flow instability and forced convection in fluid flow through rectangular and elliptical curved ducts.- Heat Transfer Engineering, vol.34, No.14, pp.1237-1248. https://doi.org/10.1080/01457632.2013.777249

[5] Dean W.R. (1927): Note on the motion of fluid in a curved pipe.- Philos Mag., vol.4, pp.208-23. https://doi.org/10.1080/14786440708564324

[6] Mondal R.N., Kaga Y., Hyakutake T. and Yanase S. (2007): Bifurcation diagram for two-dimensional steady flow and unsteady solutions in a curved square duct.- Fluid Dynamics Research, vol.39, No.5, pp.413-446. https://doi.org/10.1016/j.fluiddyn.2006.10.001

[7] Sultana M.N., Hasan M.S. and Mondal R.N. (2019): A numerical study of unsteady heat and fluid flow through a rotating curved channel with variable curvature.- AIP Conference Proceedings, vol.2121, Article No.030009. https://doi.org/10.1063/1.5115854

[8] Yanase S., Kaga Y. and Daikai R. (2002): Laminar flow through a curved rectangular duct over a wide range of aspect ratio.- Fluid Dynamics Research, vol.31, No.3, pp.151-83. https://doi.org/10.1016/S0169-5983(02)00103-X 
[9] Dolon S.N., Hasan M.S., Ray S.C. and Mondal R.N. (2019): Vortex-structure of secondary flows with effects of strong curvature on unsteady solutions through a curved rectangular duct of large aspect ratio.- AIP Conference Proceedings, vol.2121, Article No.050004. https://doi.org/10.1063/1.5115891

[10] Dolon S.N., Hasan M.S., Lorenzini, G. and Mondal R.N. (2021): A computational modeling on transient heat and fluid flow through a curved duct of large aspect ratio with centrifugal instability.- The European Physical Journal Plus, vol.136, Article No.382. https://doi.org/10.1140/epjp/s13360-021-01331-0

[11] Lin T.S., Rogers S., Tseluiko D. and Thiele U. (2016): Bifurcation analysis of the behavior of partially wetting liquids on a rotating cylinder.- Physics of Fluids, vol.28, Article No.082102. https://doi.org/10.1063/1.4959890

[12] Watanabe T. and Yanase S. (2013): Bifurcation study of three-dimensional solutions of the curved square-duct flow.- Journal of the Physical Society of Japan, vol.82, Article No.074402. https://doi.org/10.7566/JPSJ.82.074402

[13] Hasan M.S., Rashid S., Dolon S.N., Chanda R.K., Islam M.M., Mondal R.N. and Lorenzini G. (2019): Investigation on energy distribution in steady and unsteady flow instabilities through a bend square pipe.- Reports in Mechanical Engineering, vol.2, No.1, pp.86-104. https://doi.org/10.31181/rme200102086h

[14] Hasan M.S., Mondal R.N. and Lorenzini G. (2020): Coriolis force effect in steady and unsteady flow characteristics with convective heat transfer through a curved square duct.- International Journal of Mechanical Engineering, vol.5, No.1, pp.1-40.

[15] Islam M.N., Ray S.C., Hasan M.S. and Mondal R.N. (2019): Pressure-driven flow instability with convective heat transfer through a rotating curved rectangular duct with differentially heated top and bottom walls.- AIP Conference Proceedings, vol.2121, Article No.030011. https://doi.org/10.1063/1.5115856

[16] Zhang W., Wei Y., Dou H.S. and Zhu Z. (2018): Transient behaviors of mixed convection in a square enclosure with an inner impulsively rotating circular cylinder.- International Communications in Heat and Mass Transfer, vol.8, pp.143-154. https://doi.org/10.1016/j.icheatmasstransfer.2018.08.016

[17] Mondal R.N., Kaga Y., Hyakutake T. and Yanase S. (2006): Effects of curvature and convective heat transfer in curved square duct flows.- Trans. ASME, Journal of Fluids Engineering, vol.128, No.9, pp.1013-1022. https://doi.org/10.1115/1.2236131

[18] Mondal R.N., Islam M.S., Uddin K. and Hossain M.A. (2013): Effects of aspect ratio on unsteady solutions through curved duct flow.- Applied Mathematics and Mechanics, vol.34, No.9, pp.1107-1122. https://doi.org/10.1007/s10483-013-1731-8

[19] Tsuda N. and Ohba K. (1984): Laser Doppler measurements of developing laminar flow in a moderately curved Ubend of square cross section.- Proceedings of the Second Osaka Symposium on Flow Measuring Techniques, Osaka, Japan.

[20] Wang L., Pang O. and Cheng L. (2005): Bifurcation and stability of forced convection in tightly coiled ducts: multiplicity.- Chaos, Solitons and Fractals, vol.26, pp.337-352. https://doi.org/10.1016/j.chaos.2004.12.026

[21] Islam M.Z., Mondal R.N. and Rashidi M.M. (2017): Dean-Taylor flow with convective heat transfer through a coiled duct.- Computers and Fluids, vol.149, pp.41-55. https://doi.org/10.1016/j.compfluid.2017.03.001

[22] Hasan M.S., Mondal R.N. and Yanase S. (2021): Numerical prediction of unsteady fluid flow and heat transfer through a stationary curved square duct.- AIP Conference Proceedings, vol.2324, Article No.050020. https://doi.org/10.1063/5.0037783

[23] Hasan M.S., Chanda R.K., Mondal R.N. and Lorenzini G. (2019): Effects of rotation on unsteady fluid flow and forced convection in the rotating curved square duct with a small curvature.- Facta Universitatis, Series: Mechanical Engineering, accepted. https://doi.org/10.22190/FUME210129041A

[24] Hasan M.S., Islam M.S., Badsha M.F., Mondal R.N. and Lorenzini G. (2020): Numerical investigation on the transition of fluid flow characteristics through a rotating curved duct.- International Journal of Applied Mechanics and Engineering, vol.25, No.3, pp.45-63. https://doi.org/10.2478/ijame-2020-0034

[25] Helal M.N.A., Ghosh B.P. and Mondal R.N. (2016): Numerical simulation of two-dimensional laminar flow and heat transfer through a rotating curved square channel.- American Journal of Fluid Dynamics, vol.6, No.1, pp.110. https://doi.org/10.5923/j.ajfd.20160601.01

[26] Yanase S., Watanabe T. and Hyakutake T. (2008): Traveling-wave solutions of the flow in a curved-square duct.Physics of Fluids, vol.20, Article No.124101. https://doi.org/10.1063/1.3029703

[27] Yamamoto K., Wu X., Hyakutake T. and Yanase S. (2004): Taylor-Dean flow through a curved duct of square cross section.- Fluid Dynamics Research, vol.35, pp.67-86. https://doi.org/10.1016/j.fluiddyn.2004.04.003 
[28] Chandratilleke T.T. and Nursubyakto. (2003): Numerical prediction of secondary flow and convective heat transfer in externally heated curved rectangular ducts.- International Journal of Thermal Sciences, vol.42, pp.187-198. https://doi.org/10.1016/S1290-0729(02)00018-2

[29] Mondal R.N., Watanabe T., Hossain M.A. and Yanase S. (2017): Vortex-structure and unsteady solutions with convective heat transfer trough a curved duct.- Journal of Thermophysics And Heat Transfer, vol.31, No.1, pp.243-253. https://doi.org/10.2514/1.T4913

[30] Ray S., Hasan M.S. and Mondal R.N. (2020): On the onset of hydrodynamic instability with convective heat transfer through a rotating curved rectangular duct.- Mathematical Modelling of Engineering Problems, vol.7, No.1, pp.31-44. https://doi.org/10.18280/mmep.070105

[31] Chandratilleke T.T., Nadim N. and Narayanaswamy R. (2012): Vortex structure-based analysis of laminar flow behaviour and thermal characteristics in curved ducts.- International Journal of Thermal Sciences, vol.59, pp.7586. https://doi.org/10.1016/j.ijthermalsci.2012.04.014

[32] Datta A.K., Hayamizu Y., Kouchi T. and Nagata Y., Yamamoto K. and Yanase S. (2017): Numerical study of turbulent helical pipe flow with comparison to the experimental results.- Journal of Fluids Engineering, vol.139, Article No.091204. https://doi.org/10.1115/1.4036477

[33] Wang L.Q. (1997): Buoyancy-force-driven transitions in flow structures and their effects on heat transfer in a rotating curved channel.- International Journal of Heat Mass Transfer, vol.40, No.2, pp.223-235. https://doi.org/10.1016/0017-9310(96)00127-5

[34] Razavi S.E., Soltanipour H. and Choupani P. (2015): Second law analysis of Laminar Forced Convection in a rotating curved duct.- Thermal Sciences, vol.19, No.1, pp.95-107. https://doi.org/10.2298/TSCI120606034R

[35] Hasan M.S., Mondal R.N., and Lorenzini G. (2019): Centrifugal-Coriolis instability through a rotating curved square duct with bottom wall heating and cooling from the ceiling.- AIP Conference Proceedings, vol.2324, Article No.040007. https://doi.org/10.1063/5.0037784

[36] Hasan M.S., Dolon S.N., Chakraborty H.S., Mondal R.N. and Lorenzini G. (2021): numerical investigation on flow transition through a curved square duct with negative rotation.- Journal of Applied and Computational Mechanics, vol.7, No.3, pp.1435-1447. https://doi.org/10.22055/JACM.2020.33606.2253

[37] Hasan M.S., Mondal R.N. and Lorenzini G. (2020): Physics of bifurcation of the flow and heat transfer through a curved duct with natural and forced convection.- Chinese Journal of Physics, vol.67, pp.428-457. https://doi.org/10.1016/j.cjph.2020.07.004

[38] Promvonge P. and Skullong S. (2020): Thermo-hydraulic performance in heat exchanger tube with V-shaped winglet vortex generator.- Applied Thermal Engineering, vol.164, Article No.114424. https://doi.org/10.1016/j.applthermaleng.2019.114424

[39] Norouzi M., Kayhani M.H., Nobari M.R.H. and Demneh M.K. (2009): Convective heat transfer of viscoelastic flow in a curved duct.- World Academy of Science, Engineering and Technology, International Journal of Mechanical and Mechatronics Engineering, vol.3, No.8, pp.921-927. https://doi.org/10.5281/zenodo.1081071

[40] Garcia F., Sanchez J., Dormy E. and Net M. (2015): Oscillatory convection in rotating spherical shells: low Prandtl number and non-slip boundary conditions.- Siam Journal on Applied Dynamical Systems, vol.14, No.4, pp.1787-1807. https://doi.org/10.1137/15M100729X

[41] Chanda R.K., Hasan M.S., Alam M.M., Mondal R.N. (2021): Taylor-heat flux effect on fluid flow and heat transfer in a curved rectangular duct with rotation.- International Journal of Applied and Computational Mathematics, vol.7, Article No.146. https://doi.org/10.1007/s40819-021-00986-8

[42] Dolon S.N., Hasan M.S., Lorenzini G. and Mondal R.N. (2021): A computational modeling on transient heat and fluid flow through a curved duct of large aspect ratio with centrifugal instability.- The European Physical Journal Plus, vol.136, Article No.382, p.27. https://doi.org/10.1140/epjp/s13360-021-01331-0

[43] Facao J. and Oliveira A.C. (2005): Modeling laminar heat transfer in a curved rectangular duct with a computational fluid dynamics code.- Numerical Heat Transfer, Part A, vol.48, pp.165-177. https://doi.org/10.1080/10407780590948918

[44] Gottlieb D. and Orazag S.A. (1977): Numerical Analysis of Spectral Methods.- Society of Industrial and Applied Mathematics, Philadelphia, USA. https://doi.org/10.1137/1.9781611970425

[45] Mees P.A.J., Nandakumar K. and Masliyah J.H. (1996): Instability and transitions of flow in a curved square duct: the development of the two pairs of Dean vortices.- Journal of Fluid Mechanics, vol.314, pp.227-246. https://doi.org/10.1017/S0022112096000298 
[46] Wang L. and Yang T. (2005): Periodic oscillation in curved duct flows.- Physica D., vol.200, pp.296-302. https://doi.org/10.1016/j.physd.2004.11.003

[47] Yamamoto K., Wu X., Nozaki K. and Hayamizu Y. (2006): Visualization of Taylor-Dean flow in a curved duct of square cross-section.- Fluid Dynamics Research, vol.38, pp.1-18. https://doi.org/10.1016/j.fluiddyn.2005.09.002

[48] Bara B., Nandakumar K. and Masliyah J.H. (1992): An experimental and numerical study of the Dean problem: flow development towards two-dimensional multiple solutions.- Journal of Fluid Mechanics, vol.244, pp.339-76. https://doi.org/10.1017/S0022112092003100

Received: February 24, 2021

Revised: August 9, 2021 